\title{
Fault Diagnosis Using a Timed Discrete Event Approach Based on Interval Observers: Application to Sewer Networks
}

\author{
Jordi Meseguer, Vicenç Puig and Teresa Escobet
}

\begin{abstract}
This paper proposes a fault diagnosis method using a timed discrete-event approach based on interval observers which improves the integration of fault detection and isolation tasks. The interface between fault detection and fault isolation considers the activation degree and the occurrence time instant of the diagnostic signals using a combination of several theoretical fault signature matrices which store the knowledge of the relationship between diagnostic signals and faults. The fault isolation module is implemented using a timed discrete event approach that recognizes the occurrence of a fault by identifying a unique sequence of observable events (fault signals). The states and transitions that characterize such a system can be inferred directly from the relation between fault signals and faults. The proposed fault diagnosis approach has been motivated by the problem of detecting and isolating faults of the Barcelona's urban sewer system limnimeters (level meter sensors). The results obtained in this case study illustrate the benefits of using the proposed approach in comparison with the standard fault detection and isolation approach.
\end{abstract}

Index Terms - Fault Detection, Fault Diagnosis, Robustness, Observers, Intervals, Discrete-event Systems.

\section{INTRODUCTION}

$\mathrm{T}$ HIS paper proposes a fault diagnosis method based on a timed discrete-event approach using interval observers to solve the problem of fault detection and isolation (FDI) in the Barcelona sewer network. This network has a telemetry system used in real-time for the control system. Sewer networks are complex large-scale systems which in turn require highly sophisticated supervisory-control systems to ensure that high performance can be achieved and maintained

Manuscript received XXXXX. The authors wish also to thank the support received by the by CICYT (ref. WATMAN DPI2009-13744) and (ref. HYFA DPI2008-01996) of Spanish Ministry of Education.

J. Meseguer is with the Advanced Control Systems (SAC) Research Group, Universitat Politècnica de Catalunya (UPC), Rambla Sant Nebridi 10, 08222 Terrassa (Spain). (e-mail: jordimeseguer@hotmail.com)

V. Puig is with the Advanced Control Systems (SAC) Research Group and with the Institut de Robòtica i Informàtica Industrial (CSIC), Universitat Politècnica de Catalunya (UPC), Llorenç i Artigas 4-6, 08028 Barcelona (Spain). (Phone: +34 73998627, Phone: +34 7398826, e-mail: vicenc.puig@upc.edu).

T. Escobet is with the Advanced Control Systems (SAC) Research Group, Universitat Politècnica de Catalunya (UPC), Rambla Sant Nebridi 10, 08222 Terrassa (Spain). (e-mail: teresa.escobet@upc.edu). under adverse conditions. Most cities around the world have sewage systems that combine sanitary and storm water flows within the same network. This is why these networks are known as Combined Sewage Systems (CSS). During rain storms, wastewater flows can easily overload these CSS, thereby causing operators to dump the excess of water into the nearest receiver environment (rivers, streams or sea). This discharge to the environment, known as Combined Sewage Overflow (CSO), contains biological and chemical contaminants creating a major environmental and public health hazard. Environmental protection agencies have started forcing municipalities to find solutions in order to avoid those CSO events. A possible solution to the CSO problem would be to enhance existing sewer infrastructure by increasing the capacity of the wastewater treatment plants (WWTP) and by building new underground retention tanks. But in order to take profit of these expensive infrastructures, it is also necessary a highly sophisticated real-time control (RTC) scheme which ensures that high performance can be achieved and maintained under adverse meteorological conditions [17]. In particular, the optimal real-time global control of a Barcelona sewer network aims to minimise flooding and combined sewer overflow to the environment, and to maximise the wastewater treatment plants utilisation. However, the global optimal control of the sewer network is vulnerable to faults. Faults in sensors (rain-gauges and limnimeters) and actuators (gates and pumps), specially in heavy rain scenarios, are usual. If these faults were not detected and isolated, the global optimal control would derive in anomalous performance of the network system, being necessary to move the control to the local mode ${ }^{1}$. This will make very difficult the success of the global control system. One way of achieving fault-tolerance is to employ an on-line FDI scheme, such that when a fault is detected and isolated, it will activate in response some accommodation action, which can be pre-determined for each fault.

In general, when addressing the problem of FDI, two strategies can be found in the literature: hardware redundancy

When local control is applied, flow regulation devices use only measurements taken at their specific locations. On the other hand, when global control is used, control actions are computed taking into account real-time measurements all through the network, using in the best possible the infrastructure capacity and all the available sensor information. 
based on the use of redundant (extra) sensors and software (or analytical) redundancy based on the use of a mathematical model that combines measurements from other sensors or from the same sensor in past instants [23]. In critical systems (space aircrafts, aeroplanes, ...), hardware redundancy is preferred. But, for large scale systems (as the case of sewer networks), the use of hardware redundancy is very expensive and increases the number of maintenance and calibration operations. This is the reason why analytical redundancy has been recognized to be a good and cheaper alternative. This is the approach followed in this paper. In the literature, some related works of sensor fault diagnosis in sewer networks can be found that address the case of rain-gauges [38] or the case of limnimeters [2][3] .

At the beginning of the research presented in this paper, the FDI problem in sewer network limnimeters was addressed using the standard well established fault diagnosis methodologies coming from FDI community [12]. However, several deficiencies were detected either in the fault detection or isolation stages when the standard FDI theory was applied to solve this problem. Mainly, the deficient performance was due to the poor (binary) interface between fault detection and fault isolation and the no-consideration of the fault signal dynamics, as pointed out by [5] and [27]. This have motivated the development of the model based fault diagnosis methodology presented in this paper based on the use of interval observers (in order to enhance robustness against modelling errors) in fault detection and on the use of a timed discrete-event isolation algorithm based on several fault signature matrices that considers additional information to the typical binary one. In particular, fault signature matrices containing information about residual fault sensitivity and residual time/order activation are used. Thereby, in the proposed approach, fault signals are represented as a temporal sequence of discrete events using a qualitative approach while fault detection is based on an analytical model represented by an interval observer which takes account the parametric model uncertainty. This is why this approach can be considered as a BRIDGE approach [1] that tries to benefit from the best of the $F D I$ and $D X$ diagnosis communities.

The structure of the paper is the following: a description of the case study is given in Section II. Then, in Section III, the motivation and overview of the new fault diagnosis approach are introduced. The fault detection using interval observers is introduced in Section $I V$ focusing on the generation of fault signals and on their connection with the residual generator structure: the case of study is used to exemplify the obtained results. Next, in Section $V$, the interface between fault detection and fault isolation is presented showing how to obtain the theoretical fault signature matrices. In Section VI, the fault isolation algorithm based on a timed discrete event system is exposed. The interval observer-based fault diagnosis algorithm will be applied to different fault scenarios of the case study to assess the validity of the derived results in Section VII. Section VIII closes the paper with the main conclusions.

\section{Description OF THE CASE StUdy}

\section{A. Barcelona Sewer Network Description}

The city of Barcelona, with a population of 3,000,000 inhabitants in an area of 98 square $\mathrm{Km}^{2}$, has a combined sewer system (waste and rainwater go into the same sewers) of approximately $1,500 \mathrm{Km}$. Additionally, the yearly rainfall is not very high $(600 \mathrm{~mm} /$ year$)$, but it includes heavy storms typical of the Mediterranean climate that cause a lot of flooding problems and combined sewer overflows to the sea that cause pollution. Such a complex system is conducted through the control centre in CLABSA (Barcelona Sewer Company) using a remote control system (in operation since 1994) that includes sensors, regulators, remote stations and communications (Fig. 1). Nowadays, for control purposes, the urban drainage system contains 21 pumping stations, 36 gates, 10 valves and 10 detention tanks which are regulated in order to prevent flooding and combined sewer overflow to the environment. The remote control system is equipped with 56 remote stations including 22 rain-gauges and 136 water-level sensors which provide real-time information about rainfall and water levels into the sewer system. All this information is centralized at the CLABSA Control Center through a supervisory control and data acquisition (SCADA) system. In local mode, all regulated elements (pumps, gates and detention tanks) are controlled locally, i.e., they are handled from the remote control centre according to the measurements of sensors connected only to the local station.

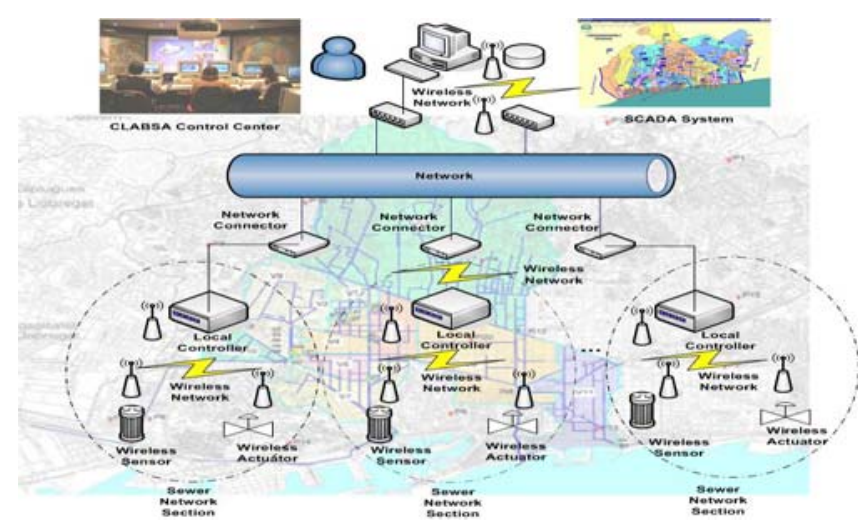

Fig. 1. Scheme of the mechanical treatment unit to grind and classify a mineral flow

In global mode, the real-time control of the sewer network is based on model predictive control (MPC) which sets the references for local controllers located on different actuator (gates and pumps) elements of the sewer network using measurements taken from sensors distributed along the network and rain sensors. These references are computed in real-time using an operational model to predict time ahead the network dynamics, the current state of the system, provided by sensors, the current rain intensity measurements and appropriate rainfall predictions [4]. The control objective is to minimize flooding and combined sewer overflow to the environment, and to maximize the utilization of wastewater 
treatment plants. CORAL is the application software developed to implement in real-time the optimal MPC global control of the Barcelona sewer network (Fig. 2). This application has been jointly developed by the SAC research group at UPC and CLABSA. It is built in three main modules: the first one is the model manager, which is the tool used to define a model and all its parameters. The second one is the event simulator and reproducer, which is used to evaluate the system configuration and control strategies, to read a past rain from CORAL database and to display the consequent system behaviour against rain scenarios. Finally, the third one is the online controller, which is used to read all sensor and actuator data from the SCADA system, link all this data to the optimizer and launching it to calculate/compute the optimal control strategy and finally, send this strategy in form of actuator set-points to the SCADA. More details about this tool can be found in [31].

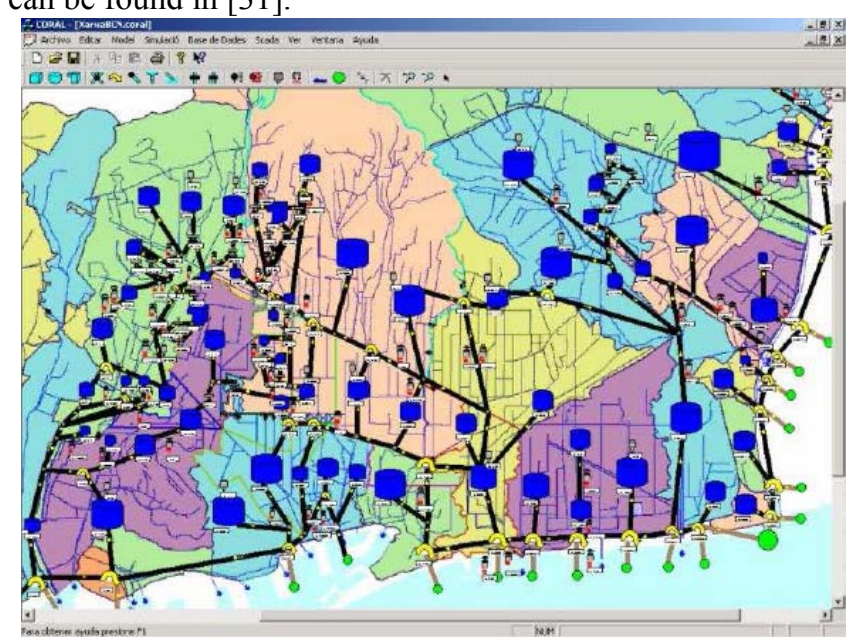

Fig. 2. CORAL: MPC control tool for sewer networks

\section{B. Case Study Description}

The case of study used to illustrate the fault diagnosis methodology proposed in this paper is based on a part of this network that covers a surface of $22,6 \mathrm{Km}^{2}$. It is constituted by 12 catchments (shaded areas of Figure 3) of the twenty catchments of the city and contains 3 diversion gates, 5 overflows and only one real detention tank, whose capacity is $35000 \mathrm{~m}^{3}$. It includes the main sewer which carries water to the treatment plants and the four main seafront sea pollution points. Moreover, in this part of the network there are five passive flow-diversion (overflow) devices and the network is metered by means of 4 rain-gauges and 14 limnimeters.

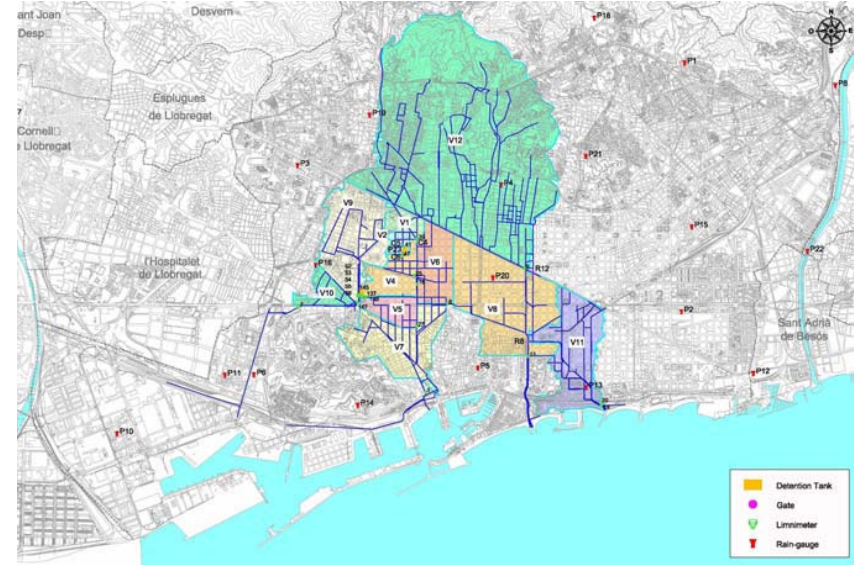

Fig. 3. Part of Barcelona network considered in the limnimeters FDI case study

The water flow in sewers due to runoff is modeled as an open-channel and can be described accurately using SaintVenant equations $^{2}$ [17], obtaining a complex non-line rainfallrunoff model that is being used for high-fidelity simulations. However, for on-line purposes, as the global optimal control and FDI, a simpler model must be used. The method used to derive a rainfall-runoff real-time model of a sewer network is through a simplified graph relating the main sewers and catchments as a set of virtual reservoirs [4]. A virtual reservoir is a conceptual model of a sewer network catchment that approximates the hydraulics of the retention of rain, runoff and sewage water. The hydraulics of a virtual reservoir can be described by the following equation:

$$
\frac{d V(t)}{d t}=Q_{u p}(t)-Q_{d o w n}(t)+P(t) S
$$

where: $V$ is the water volume accumulated in the catchment, $Q_{u p}$ and $Q_{\text {down }}$ are flows entering and exiting the catchment, $P$ is the rain intensity falling in the catchment and $S$ its surface (Fig. 4).

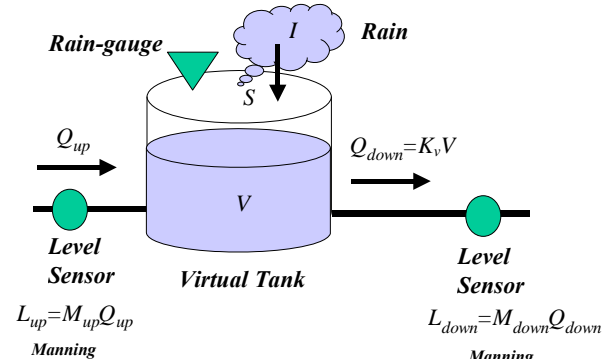

Fig. 4. Virtual model of a sewer catchment

Input and output sewer levels are measured using limnimeters and they can be associated with flows using a linearised Manning relation calibrated experimentally [10], as follows: $\quad Q_{u p}(t)=M_{u p} L_{u p}(t) \quad$ and

\footnotetext{
2 Saint-Venant equations are based on physical principles of mass conservation and energy, allow to describe accurately the open-channel flow in a sewer. However, these partial differential equations can only be solved numerically using computer intensive methods.
} 
$Q_{\text {down }}(t)=M_{\text {down }} L_{\text {down }}(t)$, where $M$ is the Manning coefficient that relates the flow value in the sewer with the limnimeter measurement and $L_{\text {up }}, L_{\text {down }}$ are the measured levels. Moreover, assuming that the catchments behaves as linear virtual tanks: $Q_{\text {down }}(t)=K_{v} V(t)$, where $K_{v}$ is defined as the conversion coefficient from volume to flow. Then, substituting these relations in (1) and considering that the measurement sampling time $\left(T_{s}=300 \mathrm{~s}\right.$ in the Barcelona network), the following discrete-time model relating the liminimeter measurements of a given virtual reservoir can be derived:

$$
L_{\text {down }}(k+1)=a_{\text {down }} L_{\text {down }}(k)+a_{\text {up }} L_{\text {up }}(k)+b P(k)
$$

where: $a_{\text {down }}=\left(1-K_{v} T_{s}\right), \quad a_{u p}=K_{v} T_{s} M_{u p} / M_{\text {down }}$ and $b=S K_{v} T_{s} / M_{\text {down }}$.

Using this modelling methodology, the case of study model is presented in Fig. 5.

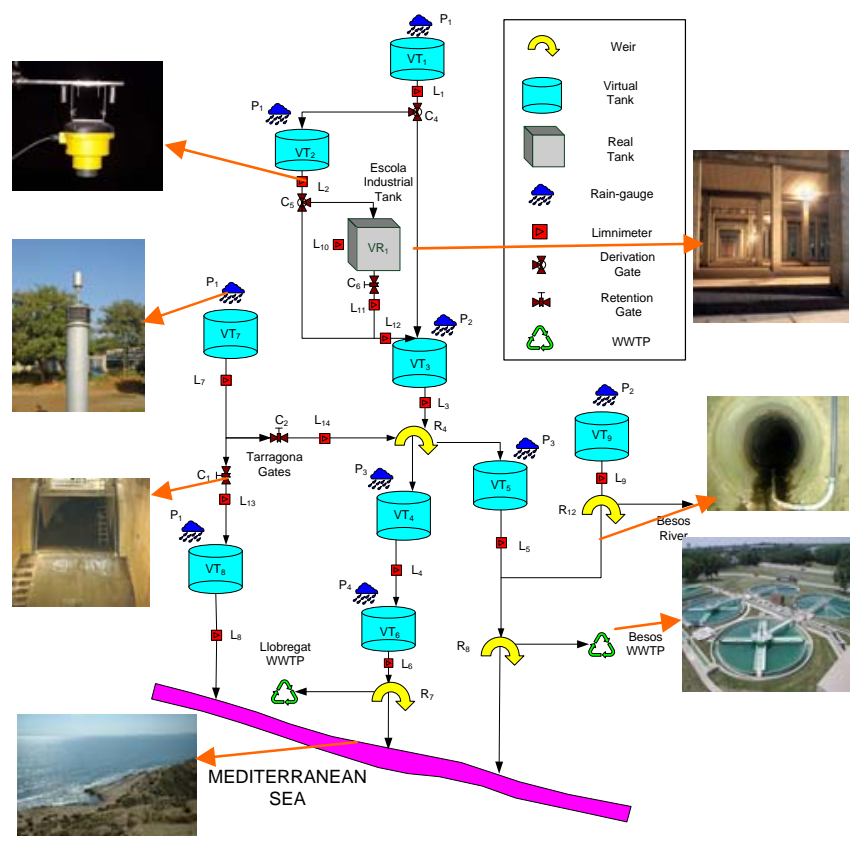

Fig. 5. Virtual reservoir model of the Barcelona sewer network case study

In this part of the network there are: 14 limnimeters $\left(L_{1}, \ldots, L_{14}\right)$ and 4 rain-gauges $\left(P_{1}, \ldots, P_{4}\right)$. Rain-gauges are spatially distributed in Barcelona area and each one affects more than one catchment (see Fig. 5). Applying (2) to each virtual reservoir considering the main paths, a set of 9 discrete-time equations can be obtained and can be expressed as follows:

$$
\hat{L}_{i}(k+1)=a_{i, i} L_{i}(k)+\sum_{\substack{j \neq i \\ j=1}}^{9} a_{i, j} L_{j}(k)+\sum_{q=1}^{4} b_{i, q} P_{q}(k) \quad i=1, \ldots, 9
$$

where following model (2): $a_{i, i}$ is computed as $a_{\text {down }} ; a_{i, j}$ is 0 if the tank $j$ is not a preceding of tank $i$, otherwise the value is computed as $a_{u p} ; b_{i, q}$ is 0 if tank $j$ is not affected by $P_{q}$, otherwise the value is computed as $b$. Then, Eq. (3) can also be written in discrete-time space state by defining the volumes of the virtual reservoirs as state variables $(x)$, the limnimeters as output variables $(y)$ and the rain-gauges as input variables (u).

The model related to the case of study has an additional equation corresponding to the real tank relating the tank water level with the input and output flows

$$
\hat{L}_{10}(k+1)=L_{10}(k)+T_{s}\left(M_{2} L_{2}(k)-M_{11} L_{11}(k)\right)
$$

Moreover, two additional static mass balance equations can be written relating the flows measured by limnimeters: $L_{11}$ and $L_{12}$, and by $L_{7}, L_{13}$ and $L_{14}$, respectively.

As a result of applying the limnimeter model methodology described above, 12 analytical redundancy relations are obtained [37]. Table I presents the structural analysis of these relations. Each row is a redundant relation, denoted by $r_{i}$, and each column is related to the variable measurements involved, either, limnimeters or rain-gauges. Thereby, a cross in a given cell indicates that the corresponding variable measurement is present in the related relation.

TABLE I

STRUCTURAL ANALYSIS OF THE LIMNIMETER MODELS ASSOCIATED WITH THE CASE OF STUDY

\begin{tabular}{|l|l|l|l|l|l|l|l|l|l|l|l|l|l|l|l|l|l|l|}
\hline & $L_{1}$ & $L_{2}$ & $L_{3}$ & $L_{4}$ & $L_{5}$ & $L_{6}$ & $L_{7}$ & $L_{8}$ & $L_{9}$ & $L_{10}$ & $L_{11}$ & $L_{12}$ & $L_{13}$ & $L_{14}$ & $P_{1}$ & $P_{2}$ & $P_{3}$ & $P_{4}$ \\
\hline$r_{1}$ & $\mathrm{X}$ & & & & & & & & & & & & & & $\mathrm{X}$ & & & \\
\hline$r_{2}$ & $\mathrm{X}$ & $\mathrm{X}$ & & & & & & & & & & & & & $\mathrm{X}$ & & & \\
\hline$r_{3}$ & & & $\mathrm{X}$ & & & & & & & & & $\mathrm{X}$ & & & & $\mathrm{X}$ & & \\
\hline$r_{4}$ & & & & $\mathrm{X}$ & & & & & & & & & & & & & $\mathrm{X}$ & \\
\hline$r_{5}$ & & & $\mathrm{X}$ & & $\mathrm{X}$ & & $\mathrm{X}$ & & & & & & & $\mathrm{X}$ & & & $\mathrm{X}$ & \\
\hline$r_{6}$ & & & & $\mathrm{X}$ & & $\mathrm{X}$ & & & & & & & & & & & & $\mathrm{X}$ \\
\hline$r_{7}$ & & & & & & & $\mathrm{X}$ & & & & & & & & $\mathrm{X}$ & & & \\
\hline$r_{8}$ & & & & & & & & $\mathrm{X}$ & & & & & $\mathrm{X}$ & & $\mathrm{X}$ & & & \\
\hline$r_{9}$ & & & & & & & & & $\mathrm{X}$ & & & & & & & $\mathrm{X}$ & & \\
\hline$r_{10}$ & & $\mathrm{X}$ & & & & & & & & $\mathrm{X}$ & $\mathrm{X}$ & & & & & & & \\
\hline$r_{11}$ & & & & & & & & & & & $\mathrm{X}$ & $\mathrm{X}$ & & & & & & \\
\hline$r_{12}$ & & & & & & & $\mathrm{X}$ & & & & & & $\mathrm{X}$ & $\mathrm{X}$ & & & & \\
\hline
\end{tabular}

To exemplify the proposed FDI methodology, fault scenarios presented in this paper are focused on detecting and locating faults affecting the limnimeters that appear in the case study presented in Fig. 5. Thus, the vector of faults $f_{y}$ to be detected is

$$
\boldsymbol{f}_{y}=\left[f_{L_{1}}, \ldots, f_{L_{14}}\right]^{T}
$$

It is assumed that just a single fault appears in a certain faulty scenario and faults in limnimeters can be modelled additively as usually done in the case of sensors in the FDI approach [12].

Conversely, it can be noticed that Table I, which determines the limnimeter model structure, also includes rain-gauges. However, in [26], it is shown that faults in rain-gauges can be handled independently from faults in liminimeters ${ }^{3}$. The complete FDI approach with faults in rain-gauges and actuators (valves and pumps) is presented in [30].

\footnotetext{
3 Rain-gauge analytical redundancy relations can be derived relating the most correlated rain-gauges, derived using statistical tools that allow exploiting the spatial redundancy in the rain-gauge network. Thus, those relations allow to detect and isolate faults in rain-gauges independently of faults in limnimeters. When a rain-gauge is affected by a fault, it is replaced by the most correlated rain-gauges.
} 


\section{OVERVIEW OF THE PROPOSED APPROACH}

\section{A. Evaluation of the Existing FDI Methods}

Model-based fault detection and isolation is based on a certain set of numerical fault indicators, known as residuals $\boldsymbol{r}(k)$ which are computed using the measured inputs $\boldsymbol{u}(k)$ and outputs $\boldsymbol{y}(k)$ of the monitored system:

$$
\boldsymbol{r}(k)=\boldsymbol{\Psi}(\boldsymbol{y}(k), \boldsymbol{u}(k))
$$

where $\boldsymbol{\Psi}$ is the residual generator function. This function allows computing the residual set at every time instant using the measurements of the system inputs and outputs. Ideally, according to [12], residuals should be zero (or less than a threshold that takes into account noise and model uncertainty) when no fault is affecting the system. The fault detection task consists in deciding if there is a fault affecting the monitored system by checking each residual $r_{i}(k)$ of the residual set against a threshold that takes into account model uncertainty, noise and the unknown disturbances. The result of this test applied to every residual $r_{i}(k)$ produces an observed fault signature $\phi(k): \phi(k)=\left[\phi_{1}(k), \phi_{2}(k), \cdots, \phi_{n_{\phi}}(k)\right]$. A basic way of obtaining these observed fault signals could be through a binary evaluation of every residual $r_{i}(k)$ against a threshold $\tau_{i}$ [12]:

$$
\phi_{i}(k)=\left\{\begin{array}{lll}
0 & \text { if } & \left|r_{i}(k)\right| \leq \tau_{i} \\
1 & \text { if } & \left|r_{i}(k)\right|>\tau_{i}
\end{array}\right.
$$

The observed fault signature is, then, supplied to the fault isolation module that will try to isolate the fault so that a fault diagnosis can be given. This module is able to produce such a fault diagnosis since it has the knowledge about the binary relation between the considered fault hypothesis set $\boldsymbol{f}(k)=\left\{f(k)_{1}, f_{2}(k), \cdots, f_{n_{f}}(k)\right\}$ and the fault signal set $\phi(k)$. This relation is stored in the called theoretical binary fault signature matrix (FSM). Thereby, an element $F S M_{i j}$ of this matrix is equal to 1 if the fault hypothesis $f_{j}(k)$ is expected to affect the residual $r_{i}(k)$ such that the related fault signal $\phi_{i}(k)$ is equal to 1 when this fault is affecting the monitored system. Otherwise, the element $F S M_{i j}$ is zero-valued.

However, this basic fault detection and isolation scheme, as already pointed out in the introduction, has the following drawbacks, among others (for more details see [5] and [27]):

(a) The threshold $\tau_{i}$ should be determined and adapted online according to the system inputs and outputs taking into account the model uncertainty.

(b) The presence of the noise produces chattering if a binary evaluation of the residual is used.

(c) All fault signals $\phi_{i}(k)$ affected by a certain fault $f_{j}(k)$ according to the structure of the matrix $\boldsymbol{F S M}$ should be activated at the same time instant and they should be persistently observed during the whole fault isolation process. Otherwise, a wrong fault diagnosis result could be given. Nonetheless, because fault signals have their own dynamics, neither they necessarily have to be activated at the same time nor they are persistently observed.

(d) Restricting the relation between faults and fault signals to a binary one causes a loss of useful information that can add fault distinguishability and accurateness to the fault isolation algorithm preventing possible wrong fault diagnosis results. The occurrence of a fault causes the apparition of a certain subset of fault signals such that each of them have characteristic dynamical properties for this fault which can improve the performance of the fault isolation algorithm if they are taken into account.

Some of these problems should be considered by the fault detection module (for example, (a) and (b)), while the others by the fault isolation module (for example, (c) and (d)), or by the interface between both stages.

\section{B. Proposed Approach}

To address all these problems when using the standard FDI approach to limnimeter fault detection and isolation, a new fault diagnosis approach is proposed. Fig. 6 presents an overview of the different modules and their role in such approach:

- Fault detection module generates a fault signal measuring the system inputs and outputs taking into account model uncertainty. This is carried out using a fault detection interval observer ${ }^{4}$ which allows generating an adaptive threshold that evolves along time.

Fault detection/isolation interface module evaluates fault signals generated by the fault detection module in order to register their dynamical properties which will allow the fault isolation module to isolate the fault among the considered fault hypotheses. These properties are summarized using several indicators which take into account not only the activation value of the fault signal but also its fault sensitivity/sign and its activation time/order. This improved interface module tries to handle the problems associated with the fault signal persistence, the residual sensitivity to a fault, the fault signal occurrence order and the fault signal occurrence time instant. As a result, the interface between fault detection and fault isolation modules is improved enhancing the performance of the used fault diagnosis system,

- $\quad$ Fault isolation model reasons with the information used to build all the indicators provided by the improved fault detection and isolation interface using a discrete-event fault diagnosis model that can be automatically built taking into account temporal aspects related to fault signal sequence caused by the fault (fault signal occurrence order and time instant).

${ }^{4}$ In an interval observer, model uncertainty is represented by a nominal model plus the uncertainty of every parameter bounded by intervals. 


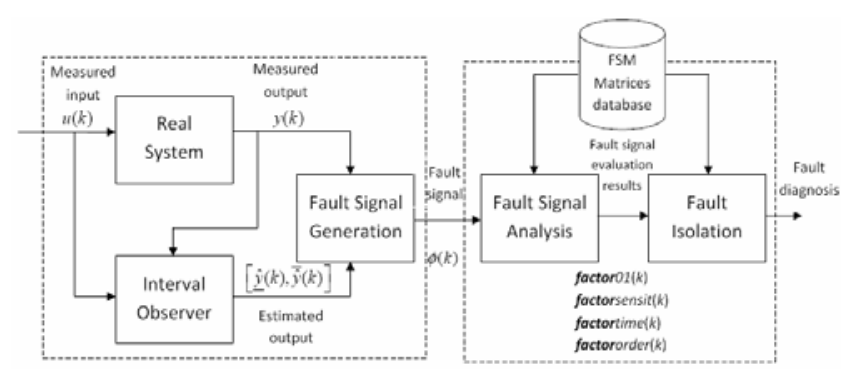

Fig. 6. Block diagram of the fault diagnosis system

\section{Fault Detection Module}

\section{A. Fault Detection Using Interval Observers}

The main purpose of the fault detection module is the generation of fault signals so that the fault can be detected. The proposed fault detection algorithm is able to handle uncertainty and consequently, it can be considered a robust approach. The robustness of a fault detection algorithm is given by the degree of sensitivity to faults compared to the degree of sensitivity to uncertainty. One of the most developed families of approaches to deal with model uncertainty, called active, is based on generating residuals, which are insensitive to uncertainty (modelling errors and disturbances), while at the same time sensitive to faults using some decoupling method [33]. On the other hand, there is a second family of approaches, called passive, which enhances the robustness of the fault detection system at the decisionmaking stage [29] using an adaptive threshold. This is the approach followed in this paper.

The FDI methodology proposed in this paper considers that the monitored system can be described analytically by a MIMO linear uncertain dynamic model in discrete-time and state-space form, including faults, as follows

$$
\begin{aligned}
& \boldsymbol{x}(k+1)=\boldsymbol{A}(\tilde{\boldsymbol{\theta}}) \boldsymbol{x}(k)+\boldsymbol{B}(\tilde{\boldsymbol{\theta}}) \boldsymbol{u}_{0}(k)+\boldsymbol{F}_{a}(\tilde{\boldsymbol{\theta}}) \boldsymbol{f}_{a}(k) \\
& \boldsymbol{y}(k)=\boldsymbol{C}(\tilde{\boldsymbol{\theta}}) \boldsymbol{x}(k)+\boldsymbol{F}_{y}(\tilde{\boldsymbol{\theta}}) \boldsymbol{f}_{y}(k)
\end{aligned}
$$

where $\boldsymbol{y}(k) \in \mathfrak{R}^{n y}, \boldsymbol{u}_{\boldsymbol{0}}(k)^{5} \in \mathfrak{R}^{n u}, \boldsymbol{x}(k) \in \mathfrak{R}^{n x}$ are the system output, input and the state-space vectors respectively; $\boldsymbol{A}(\tilde{\boldsymbol{\theta}}) \in \mathfrak{R}^{n \times \times n x}$, $\boldsymbol{B}(\tilde{\boldsymbol{\theta}}) \in \mathfrak{R}^{n x \times n u}$ and $\boldsymbol{C}(\tilde{\boldsymbol{\theta}}) \in \mathfrak{R}^{n y \times n x}$ are the state, the input and the output matrices respectively; $\tilde{\boldsymbol{\theta}}$ is the system parameter vector; $\boldsymbol{f}_{\boldsymbol{y}}(k) \in \mathfrak{R}^{n y}$ and $\boldsymbol{f}_{\boldsymbol{a}}(k) \in \mathfrak{R}^{n u}$ represent faults in the system output sensors and actuators respectively being $\boldsymbol{F}_{\boldsymbol{y}}(\tilde{\boldsymbol{\theta}}) \in \mathfrak{R}^{\text {nyxny }}$ and $\boldsymbol{F}_{\boldsymbol{a}}(\tilde{\boldsymbol{\theta}}) \in \mathfrak{R}^{n x \times n u}$ their associated matrices.

The system (7) is monitored using a linear observer with Luenberger structure based on an interval model. This type of model considers that model parameters $\boldsymbol{\theta}$ are time-invariant but bounded by an interval set $\boldsymbol{\Theta}=\left\{\boldsymbol{\theta} \in \mathfrak{R}^{n \theta} \mid \underline{\boldsymbol{\theta}} \leq \boldsymbol{\theta} \leq \overline{\boldsymbol{\theta}}\right\}$. This set represents the uncertainty about the exact knowledge of real

\footnotetext{
${ }^{5}$ It should be noticed that $\boldsymbol{u}_{0}(k)$ is the real system input and does not have to be equal to the measured system input since the input sensor might be faulty or affected by noise.
}

system parameters $\tilde{\boldsymbol{\theta}}$. The resulting interval observer can be written as:

$$
\begin{aligned}
& \hat{\boldsymbol{x}}(k+1)=(\boldsymbol{A}(\boldsymbol{\theta})-\boldsymbol{\Lambda C}(\boldsymbol{\theta})) \hat{\boldsymbol{x}}(k)+\boldsymbol{B}(\boldsymbol{\theta}) \boldsymbol{u}(k)+\boldsymbol{\Lambda} \boldsymbol{y}(k) \\
& \hat{\boldsymbol{y}}(k)=\boldsymbol{C}(\boldsymbol{\theta}) \hat{\boldsymbol{x}}(k)
\end{aligned}
$$

where $\boldsymbol{u}$ is the measured system input vector, $\hat{\boldsymbol{x}}(k)$ is the estimated system space-state vector and $\hat{\boldsymbol{y}}(k)$ is the estimated system output vector. Noticing that the relation between the measured system, $\boldsymbol{u}$, and the real system input, $\boldsymbol{u}_{\boldsymbol{0}}$, includes the effect of faults in the input sensors, the expression of $\boldsymbol{u}$ can be written as

$$
\boldsymbol{u}(k)=\boldsymbol{u}_{0}(k)+\boldsymbol{F}_{u}(\boldsymbol{\theta}) \boldsymbol{f}_{u}(k)
$$

where $\boldsymbol{f}_{\boldsymbol{u}}(k) \in \mathfrak{R}^{n u}$ is the input sensor fault while $\boldsymbol{F}_{\boldsymbol{u}}(\boldsymbol{\theta}) \in \mathfrak{R}^{\text {nuxnu }}$ is its associated matrix.

The observer gain matrix $\Lambda^{6}$ is designed to stabilize the matrix $\boldsymbol{A}_{o}(\boldsymbol{\theta})=\boldsymbol{A}(\boldsymbol{\theta})-\boldsymbol{\Lambda C}(\boldsymbol{\theta})$ and to guarantee a desired performance regarding fault detection for all $\boldsymbol{\theta} \in \boldsymbol{\Theta}$. The effect of the uncertain parameters $\boldsymbol{\theta}$ on the observer temporal response allows the interval observer (8) computing a system output interval estimation $\hat{y}(k)$ at every time instant instead of a single value. Thereby, $\hat{\boldsymbol{y}}(k)$ is bounded by the interval: $[\underline{\hat{\boldsymbol{y}}}(k), \overline{\hat{\boldsymbol{y}}}(k)]$, where for each output:

$$
\hat{y}_{i}(k)=\min _{\boldsymbol{\theta} \in \boldsymbol{\Theta}}\left(\hat{y}_{i}(k)\right) \text { and } \overline{\hat{y}}_{i}(k)=\max _{\boldsymbol{\theta} \in \boldsymbol{\Theta}}\left(\hat{y}_{i}(k)\right)
$$

Such interval can be computed using the algorithm based on numerical optimization presented in [26]. In case that there is no fault, each system output fulfils:

$$
y_{i}(k) \in\left[\underline{\hat{y}}_{i}(k), \overline{\hat{y}}_{i}(k)\right]
$$

Alternatively, the observer given by Eq. (8) can be expressed in input-output form using the $q$-transform and considering zero initial conditions as follows:

$$
\begin{aligned}
\hat{\boldsymbol{y}}(k) & =\boldsymbol{G}\left(q^{-1}, \boldsymbol{\theta}\right) \boldsymbol{u}(k)+\boldsymbol{H}\left(q^{-1}, \boldsymbol{\theta}\right) \boldsymbol{y}(k) \\
& =\boldsymbol{G}\left(q^{-1}, \boldsymbol{\theta}\right) \boldsymbol{u}_{0}(k)+\boldsymbol{H}\left(q^{-1}, \boldsymbol{\theta}\right) \boldsymbol{y}(k)+\boldsymbol{G}_{f u}\left(q^{-1}, \boldsymbol{\theta}\right) \boldsymbol{f}_{u}(k)
\end{aligned}
$$

where

$$
\begin{gathered}
\boldsymbol{G}\left(q^{-1}, \boldsymbol{\theta}\right)=\boldsymbol{C}(\boldsymbol{\theta})\left(q \boldsymbol{I}-\boldsymbol{A}_{o}(\boldsymbol{\theta})\right)^{-1} \boldsymbol{B}(\boldsymbol{\theta}) \\
\boldsymbol{H}\left(q^{-1}, \boldsymbol{\theta}\right)=\boldsymbol{C}(\boldsymbol{\theta})\left(q \boldsymbol{I}-\boldsymbol{A}_{o}(\boldsymbol{\theta})\right)^{-1} \boldsymbol{\Lambda} \\
\boldsymbol{G}_{f u}\left(q^{-1}, \boldsymbol{\theta}\right)=\boldsymbol{G}\left(q^{-1}, \boldsymbol{\theta}\right) \boldsymbol{F}_{u}(\boldsymbol{\theta})
\end{gathered}
$$

Model-based fault detection is based on generating a residual comparing the measurements of physical variables $\boldsymbol{y}(k)$ of the process with their estimation $\hat{\boldsymbol{y}}(k)$ provided by the associated system model:

$$
\boldsymbol{r}(k)=\boldsymbol{y}(k)-\hat{\boldsymbol{y}}(k)
$$

where $\boldsymbol{r}(k) \in \mathfrak{R}^{n y}$ is the residual set. According to (Gertler, 1998), a generic form of a residual generator can be obtained using Eq. (12) and written as:

\footnotetext{
${ }^{6}$ Noticing that when $\boldsymbol{\Lambda}=\mathbf{0}$, the observer is in fact a simulator but if $\boldsymbol{\Lambda} \boldsymbol{C}=\boldsymbol{A}$, the observer becomes a predictor[29].
} 


$$
\boldsymbol{r}(k)=-\boldsymbol{G}\left(q^{-1}, \boldsymbol{\theta}\right) \boldsymbol{u}(k)+\left(\boldsymbol{I}-\boldsymbol{H}\left(q^{-1}, \boldsymbol{\theta}\right)\right) \boldsymbol{y}(k)
$$

This residual expression is known as its computational form. In addition, the residual (17) can be also expressed in terms of the effects caused by faults using its internal or unknown-input-effect form [12]. This form, obtained combining (16), (12) and (7), is expressed as

$$
\begin{aligned}
\boldsymbol{r}(k) & =\boldsymbol{r}_{\theta}(k)+\left(\boldsymbol{I}-\boldsymbol{H}\left(q^{-1}, \boldsymbol{\theta}\right)\right)\left(\boldsymbol{G}_{f a}\left(q^{-1}, \tilde{\boldsymbol{\theta}}\right) \boldsymbol{f}_{a}(k)+\boldsymbol{G}_{f y}\left(q^{-1}, \tilde{\boldsymbol{\theta}}\right) \boldsymbol{f}_{y}(k)\right)+ \\
& -\boldsymbol{G}_{f u}\left(q^{-1}, \boldsymbol{\theta}\right) \boldsymbol{f}_{u}(k)
\end{aligned}
$$

where

$$
\begin{gathered}
\boldsymbol{r}_{\theta}(k)=-\boldsymbol{G}\left(q^{-1}, \boldsymbol{\theta}\right) \boldsymbol{u}_{0}(k)+\left(\boldsymbol{I}-\boldsymbol{H}\left(q^{-1}, \boldsymbol{\theta}\right)\right) \boldsymbol{y}_{0}(k) \\
\boldsymbol{G}_{f a}\left(q^{-1}, \tilde{\boldsymbol{\theta}}\right)=\boldsymbol{C}(\tilde{\boldsymbol{\theta}})(q \boldsymbol{I}-\boldsymbol{A}(\tilde{\boldsymbol{\theta}}))^{-1} \boldsymbol{F}_{a}(\tilde{\boldsymbol{\theta}}) \\
\boldsymbol{G}_{f y}\left(q^{-1}, \tilde{\boldsymbol{\theta}}\right)=\boldsymbol{F}_{y}(\tilde{\boldsymbol{\theta}})
\end{gathered}
$$

The term $\boldsymbol{r}_{\boldsymbol{\theta}}(k)$ would be the expression of the residual if the system were unaffected by faults being only caused by the parameter structured uncertainty.

Thereby, because of the consideration of model uncertainty located in the parameters as a fault detection passive robustness strategy, one way to compute the residual (18) might be using the nominal model $\hat{\boldsymbol{y}}^{o}\left(k, \boldsymbol{\theta}^{o}\right)$ given by the interval observer (8) when using $\boldsymbol{\theta}=\boldsymbol{\theta}^{\circ} \in \boldsymbol{\Theta}$.

$$
\boldsymbol{r}^{o}(k)=\boldsymbol{y}(k)-\hat{\boldsymbol{y}}^{o}(k)
$$

Then, in a non-faulty scenario, $\boldsymbol{r}^{\circ}(k)$ should be zero-valued at every time instant $k$ considering an ideal situation. Nevertheless, it will never be satisfied since the system can be affected by unknown inputs (i.e. noise, nuisance disturbances, etc.) and the model might be affected by some error assumptions (model errors) apart from its considered parameter uncertainty. Thus, the residual generated (22) can not be expected to be zero-valued in a non-faulty scenario. However, propagating the interval observer parameter uncertainty to the residual, the values of the nominal residual (22) will be bounded by the interval [29]:

$$
\left[\underline{r}_{i}^{o}(k), \bar{r}_{i}^{o}(k)\right]
$$

where:

$$
\underline{r}_{i}^{o}(k)=\underline{\hat{y}}_{i}(k)-\hat{y}_{i}^{o}(k) \text { and } \bar{r}_{i}^{o}(k)=\overline{\hat{y}}_{i}(k)-\hat{y}_{i}^{o}(k)
$$

being $\underline{y}_{i}(k)$ and $\overline{\hat{y}}_{i}(k)$ the bounds of the $i^{\text {th }}$-system output estimation computed using the interval observer (8) and obtained according to (10).

As a result, while the nominal residual $r_{i}^{o}(k)$ satisfies the following relation, a fault can not be indicated since the system outputs satisfy the relation (11).

$$
r_{i}^{o}(k) \in\left[\underline{r}_{i}^{o}(k), r_{i}^{o}(k)\right]
$$

where the interval (23) can be seen as an adaptive threshold [29]. Then, the main goal of the fault detection module consists in checking for every residual $r_{i}^{o}(k)$ if the relation (25) holds or not. According to [7], this fault detection strategy achieves robustness against model uncertainty by generating an adaptive threshold through propagating (instead of decoupling) parameter uncertainty to the residual.

\section{B. Fault Signal Generation}

The fault detection test (25) relies on the comparison of the numerical value of the nominal residual $r_{i}^{o}(k)$, which may be affected by noise, with its associated adaptive threshold. This binary procedure may lead to undesirable decision instability (chattering) because of the effect of noise on the sensor measurements and consequently, a persistency criterion should be introduced [36]. Such as indicated by the DMPapproach [24], a gradual reasoning involved by the use of fuzzy logic is an appealing alternative to bypass this chattering phenomenon. Then, as it was proposed in [26], the fault diagnostic signal (or fault signal) for each residual is calculated in the approach presented in this paper using the Kramer function [24]:

$$
\phi_{i}(k)=\left\{\begin{array}{ccc}
\frac{\left(r_{i}^{o}(k) / \bar{r}_{i}^{o}(k)\right)^{4}}{1+\left(r_{i}^{o}(k) / \bar{r}_{i}^{o}(k)\right)^{4}} & \text { if } \quad r_{i}^{o}(k) \geq 0 \\
-\frac{\left(r_{i}^{o}(k) / \underline{r}_{i}^{o}(k)\right)^{4}}{1+\left(r_{i}^{o}(k) / \underline{r}_{i}^{o}(k)\right)^{4}} \quad \text { if } & r_{i}^{o}(k)<0
\end{array}\right.
$$

The appealing performance of this function is due to its introduced grading when evaluating the residual in order to conclude the existence or not of a fault. When using the Kramer function (26), the residuals are normalized to a metric between -1 and $1, \phi_{i}(k) \in[-1,1]$, which indicates the degree of satisfaction of (25) for every nominal residual $r_{i}^{o}(k): 0$ for perfectly satisfied, 1 for severely violated high and -1 for severely violated low. In this paper, a fault signal will be notated as $\phi_{i}(k)$ and as in [14] it is considered that is activated by the fault presence if $\left|\phi_{i}(k)\right| \geq 0.5$. Otherwise, the fault signal is considered non-activated. Conversely, the set of all fault signals will be notated as $\phi=\left\{\phi_{i}: i=1,2, \ldots . . n_{y}\right\}$.

\section{Fault Signal Dynamics}

According to [12], the theoretical dynamic properties of a fault signal $\phi_{i}(k)$ caused by a given fault $f_{j}$ are set by what is known as the sensitivity of the associated residual $r_{i}(k)$ to this fault $f_{j}$. The concept of the residual sensitivity to a fault set according to [12] can be expressed analytically as

$$
\boldsymbol{S}_{\boldsymbol{f}}\left(q^{-1}\right)=\frac{\partial \boldsymbol{r}}{\partial \boldsymbol{f}}
$$

Thus, the residual sensitivity to a fault is a transfer function which describes how a fault is affecting the residual and consequently, the dynamic properties of the fault signals caused by this fault.

Concerning fault detection stage whose main task is the generation of fault signals, [21] shows the importance of this concept both in the residual time evolution and in the quality of the fault detection. 
Analyzing the residual internal form (18), and considering the fault residual sensitivity definition (27), the residual sensitivity functions to the faults $\boldsymbol{f}_{\boldsymbol{y}}, \boldsymbol{f}_{\boldsymbol{u}}$ and $\boldsymbol{f}_{\boldsymbol{a}}$ are given by the following matrices:

$$
\begin{gathered}
\boldsymbol{S}_{f y}\left(q^{-1}, \boldsymbol{\theta}\right)=\left(\boldsymbol{I}-\boldsymbol{H}\left(q^{-1}, \boldsymbol{\theta}\right)\right) \boldsymbol{G}_{f y}\left(q^{-1}, \tilde{\boldsymbol{\theta}}\right) \\
\boldsymbol{S}_{f u}\left(q^{-1}, \boldsymbol{\theta}\right)=-\boldsymbol{G}\left(q^{-1}, \boldsymbol{\theta}\right) \boldsymbol{F}_{u}(\boldsymbol{\theta}) \\
\boldsymbol{S}_{f a}\left(q^{-1}, \boldsymbol{\theta}\right)=\left(\boldsymbol{I}-\boldsymbol{H}\left(q^{-1}, \boldsymbol{\theta}\right)\right) \boldsymbol{G}_{f a}\left(q^{-1}, \tilde{\boldsymbol{\theta}}\right)
\end{gathered}
$$

where $\boldsymbol{S}_{f y} \in \mathfrak{R}^{n y} \mathrm{x} \mathfrak{R}^{n y}$ is associated with $\boldsymbol{f}_{\boldsymbol{y}}, \boldsymbol{S}_{f u} \in \mathfrak{R}^{n y} \mathrm{x} \mathfrak{R}^{n u}$ with $\boldsymbol{f}_{\boldsymbol{u}}$ and $\boldsymbol{S}_{f a} \in \mathfrak{R}^{n y} \mathrm{X} \mathfrak{R}^{n u}$ with $\boldsymbol{f}_{\boldsymbol{a}}$.

Then, taking into account (28), (29) and (30) in (18), it can be seen that this residual form can be rewritten in terms of residual sensitivity functions:

$$
\begin{aligned}
\boldsymbol{r}(k, \boldsymbol{\theta}) & =\boldsymbol{r}_{\theta}(k, \boldsymbol{\theta})+\boldsymbol{S}_{f a}\left(q^{-1}, \boldsymbol{\theta}\right) \boldsymbol{f}_{a}(k)+ \\
& +\boldsymbol{S}_{f y}\left(q^{-1}, \boldsymbol{\theta}\right) \boldsymbol{f}_{f y}(k)+\boldsymbol{S}_{f u}\left(q^{-1}, \boldsymbol{\theta}\right) \boldsymbol{f}_{f u}(k)
\end{aligned}
$$

Thereby, as the fault signals $\phi_{i}(k)$ are generated evaluating residual (31) such as indicated by (22) and (23) and applying (26), the next conclusions can be obtained:

- the fault residual sensitivity functions establish which residual $r_{i}(k)$ (fault signal $\left.\phi_{i}(k)\right)$ is affected by which fault $\left(f_{y j}, f_{u j}, f_{a j}\right)$,

- the fault residual sensitivity functions establish the dynamic of a given fault signal regarding a given fault,

- the knowledge of the dynamic properties of the fault residual sensitivity functions allow inferring the theoretical dynamic properties of the fault signal sequence generated by the occurrence of a given fault.

\section{Application to the Case Study}

The interval observer equation (12) has been applied to the network prototype presented in Section II. Notice, that (3) could be easily written as an observer model (8) where the system output, input and the state-space vectors are the measured sewer level given by the limnimeters, the rain intensity measured using rain-gauges and the predicted sewer level determined by the limnimeter model, respectively. The element of matrix $\boldsymbol{A}$ placed at the $i^{\text {th }}$-file and $j^{\text {th }}$-column is determined by the value $a_{i, j}$ of (8) while the element of matrix $\boldsymbol{B}$ placed at the $i^{\text {th }}$-file and $q^{\text {th }}$-column is given by $b_{i, q}$ of (8). These values are not null when there is a cross in the corresponding positions of Table $I$,. Concerning matrix $\boldsymbol{C}$, it is assumed to be the identity matrix. Thereby, the related limnimeters have been modelled using interval reduced observers in order to estimate their output value.

As example, in this section, the models related to limnimeters $L_{4}$ and $L_{6}$ are given:

$$
\hat{L}_{4}(k)=\frac{b_{4,3}}{1+\left(\lambda_{4,4}-a_{4,4}\right) q^{-1}} P_{3}(k)+\frac{\lambda_{4,4}}{1+\left(\lambda_{4,4}-a_{4,4}\right) q^{-1}} L_{4}(k)
$$

$$
\begin{aligned}
\hat{L}_{6}(k) & =\frac{a_{6,4}}{1+\left(\lambda_{6,6}-a_{6,6}\right) q^{-1}} L_{4}(k)+\frac{b_{6,4}}{1+\left(\lambda_{6,6}-a_{6,6}\right) q^{-1}} P_{4}(k)+ \\
& +\frac{\lambda_{6,6}}{1+\left(\lambda_{6,6}-a_{6,6}\right) q^{-1}} L_{6}(k)
\end{aligned}
$$

where $\lambda_{i, j}$ is the element of the observer gain matrix $\Lambda$ placed at the $i^{\text {th }}$-file and $j^{\text {th }}$-colum and which is parameterized as follows: $\lambda_{i, j}=w_{i, j} a_{i, j}$.

In the case of study presented in Section II, the value of the parameters and their associated uncertainty bounded using intervals are estimated using real data from the sensors installed in the network. This process guarantees that the observer estimated interval output includes all the limnimeter non-modelled effects, what is achieved using an algorithm inspired by the one proposed in [25]. This methodology uses the classical identification approaches, such as the leastsquares method, in order to provide the nominal values of the model parameters. Then, using optimization tools, the uncertainty parameter intervals of the considered reduced observer are adjusted using a worst-case approach [26] until all the measured data is covered by the model estimated output interval for the considered observer gain. Thereby, using this methodology, the parameter values of the interval reduced observer (32) and (33) are: $a_{4,4} \in$ [0.9544, 0.9737], $b_{4,3} \in[6.2641 e 3, \quad 6.3907 e 3], \quad a_{6,6} \in[0.8816, \quad 0.9084]$, $a_{6,4} \in[0.0381,0.0393]$ and $b_{6,4} \in[1.4469 e 4,1.4910 e 4]$.

In the Fig. 7, the time evolution of the measurements related to the limnimeters $L_{4}$ and $L_{6}$ and their interval estimations given by the associated interval reduced observers (32) and (33) are plotted assuming a non-faulty scenario and using the observer gains corresponding to $w_{44}=w_{66}=0.01$.
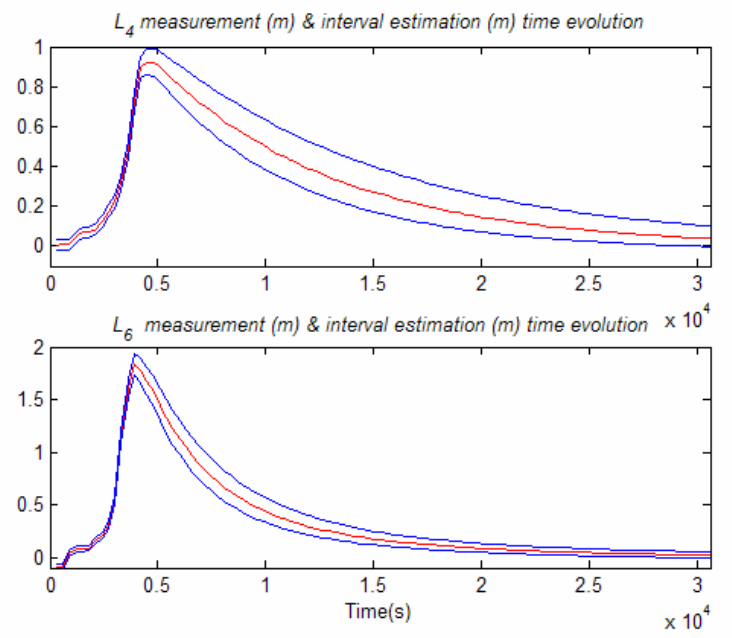

Fig. 7. Time evolution of the limnimeter output measurements and their interval estimations.

According to (16), the estimations given by the interval reduced observers related to the modelled limnimeter set (Table I) allow obtaining a set of 12 residuals. As an example, the computational form of the nominal residuals associated with the limnimeters $L_{4}$ and $L_{6}$ are given by 


$$
\begin{aligned}
r_{4}^{o}(k)= & \frac{1-a_{4,4}^{o} q^{-1}}{1+\left(\lambda_{4,4}-a_{4,4}^{o}\right) q^{-1}} L_{4}(k)-\frac{b_{4,3}^{o} q^{-1}}{1+\left(\lambda_{4,4}-a_{4,4}^{o}\right) q^{-1}} P_{3}(k) \\
r_{6}^{o}(k) & =\frac{1-a_{6,6}^{o} q^{-1}}{1+\left(\lambda_{6,6}-a_{6,6}^{o}\right) q^{-1}} L_{6}(k)-\frac{a_{6,4}^{o} q^{-1}}{1+\left(\lambda_{6,6}-a_{6,6}^{o}\right) q^{-1}} L_{4}(k)+ \\
& -\frac{b_{6,4}^{o} q^{-1}}{1+\left(\lambda_{6,6}-a_{6,6}^{o}\right) q^{-1}} P_{4}(k)
\end{aligned}
$$

where $a_{i, j}^{o}$ and $b_{i, j}^{o}$ are the nominal values of the interval parameters $a_{i, j}$ and $b_{i, j}$.

The values of $r_{4}^{o}(k)$ and $r_{6}^{o}(k)$ are bounded by the intervals $\left[\underline{r}_{4}^{o}(k), \bar{r}_{4}^{o}(k)\right]$ and $\left[\underline{r}_{6}^{o}(k), \bar{r}_{6}^{o}(k)\right]$ respectively, which are computed using the expression (24). Thereby, for the case of residual $r_{4}(k)$ :

$$
\underline{r}_{4}^{o}(k)=\underline{\hat{L}}_{4}(k)-\hat{L}_{4}^{o}(k) \text { and } \bar{r}_{4}^{o}(k)=\overline{\hat{L}}_{4}(k)-\hat{L}_{4}^{o}(k)
$$

Concerning to the residual sensitivity to a fault whose general expression is given by (27), the sensitivity of the residual $r_{4}^{o}(k)$ in (34) to an additive fault in $L_{4}\left(f_{L 4}\right)$ is given by

$$
S_{r_{4}, f_{L 4}}\left(q^{-1}\right)=\frac{1-a_{4,4}^{o} q^{-1}}{1+\left(\lambda_{4,4}-a_{4,4}^{o}\right) q^{-1}}
$$

Regarding the residual $r_{6}^{o}(k)$ in (35), its sensitivity to an additive fault in $L_{6}\left(f_{L 6}\right)$ and in $L_{4}\left(f_{L 4}\right)$ are respectively

$$
\begin{aligned}
& S_{r_{6}, f_{L 6}}\left(q^{-1}\right)=\frac{1-a_{6,6}^{o} q^{-1}}{1+\left(\lambda_{6,6}-a_{6,6}^{o}\right) q^{-1}} \\
& S_{r_{6}, f_{L 4}}\left(q^{-1}\right)=-\frac{a_{6,4}^{o} q^{-1}}{1+\left(\lambda_{6,6}-a_{6,6}^{o}\right) q^{-1}}
\end{aligned}
$$

\section{FAUlt Detection AND ISOLATION INTERFACE}

\section{A. Background}

Regarding the connection between fault detection and fault isolation modules, these stages are generally considered separately in model-based fault diagnosis (either using FDI or $D X$ methods). The typical interface between these two modules is through a binary codification of the evaluation of every residual or every analytical redundancy relation (ARR) what generates a fault signature. These last years, the integration between fault detection and fault isolation tasks in FDI model-based fault diagnosis has been a very active research area (see among others[32][35][22][15][39][40]). In this line, the dynamic properties of the fault signals caused by a certain fault depends mainly on the dynamic properties of the residual generator used in fault detection since the evaluation of this element at every time instant allows the generation of the fault signals [20]. Thus, it can be said that the fault isolation result is closely affected by the fault detection stage since the residual generator structure (used by the fault isolation module to locate the fault) determines the relationship between faults and the dynamical properties of fault signals [18]. Thus, in order to diagnose accurately, fault detection and fault isolation can not be considered independently. Besides, when observers are used as fault detection mechanism, the result of the fault detection stage is influenced by the observer gain matrix because it deeply affects the dynamic properties of the residual generator [19]. Therefore, the theoretical dynamic properties of the fault signal sequence generated by a certain fault will be also influenced by the observer gain.

In this way, it will be shown that all available and useful information of the fault detection and isolation tasks is considered. The interface between fault detection and fault isolation considers the degree of fault signal activation and the occurrence time of the diagnostic signals using a combination of several theoretical fault signature matrices which store the knowledge of the relationship between diagnostic signals and faults. Thus, the diagnosis result will be enhanced since the occurrence of a fault generates a unique sequence of observable events (fault signals) that will be recognized by the isolation module implemented as a timed discrete event system, as discussed in Section VI.

\section{B. Proposed Interface}

The used interface in this paper is based on a generalization of the theoretical fault signature matrix (FSM) [12] concept where the binary interface is extended taking into account more fault signal properties [28]. In this approach, there are as many $\boldsymbol{F S M}$ matrices as different properties are taken into account: Boolean property (FSM01), fault residual sensitivity property (FSMsensit), occurrence order property (FSMorder) and the occurrence time instant (FSMtime). Those matrices store the influence of the considered faults on the residual set: the element $\boldsymbol{F S} \boldsymbol{M}_{\mathrm{ij}}$ of a matrix contains the expected influence of fault $f_{j}$ on $r_{i}^{0}$. The following subsections illustrate how to obtain matrices FSMsensit and FSMtime using the interval observer model of the monitored system while the other two matrices can clearly be derived from them.

\section{FSMsensit: Evaluation of Fault Signal Sensitivities}

The value of an element of the table FSMsensit, FSMsensit $_{i j}$, describes how easily a fault $f_{j}\left(f_{y j}, f_{u j}, f_{a j}\right)$ will cause the $i^{\text {th }}$-residual $r_{i}^{o}(k)$ to violate its associated adaptive threshold given by the interval (23) originating the occurrence of the fault signal $\phi_{i}(k)$ (26). Thereby, according to the residual internal form (31) and the main results of Section IV.C, every element $\boldsymbol{F S M}$ sensit $_{i j}$ must be directly proportional to the fault residual sensitivity function $\boldsymbol{S}_{f}\left(q^{-1}\right)$ ((28), (29), (30) ) and inversely proportional to the associated threshold $\bar{r}_{i}^{o}(k)$ or $\underline{r}_{i}^{o}(k)$. Thereby, the following equation describes how to compute the entries $\boldsymbol{F S M}$ Sensit ij $_{i j}$ : 


$$
\text { FSMsensit }_{i j}= \begin{cases}\frac{S_{r_{i}, f_{j}}\left(q^{-1}\right) \eta\left(k-t_{0}\right)}{\left|\bar{r}_{i}^{o}(k)\right|} & \text { if } \quad r_{i}^{o}(k) \geq 0 \text { and } k \geq t_{0} \\ \frac{S_{r_{i}, f_{j}}\left(q^{-1}\right) \eta\left(k-t_{0}\right)}{\left|\underline{r}_{i}^{o}(k)\right|} & \text { if } \quad r_{i}^{o}(k)<0 \text { and } k \geq t_{0} \\ 0 \text { if } k<t_{0} \text { or } S_{r i j j}\left(q^{-1}\right)=0\end{cases}
$$

where $\eta(k)$ is an unitary abrupt step input, $S_{r_{i}, f_{j}}\left(q^{-1}\right)$ is the sensitivity associated to the nominal residual $r_{i}^{o}(k)$ regarding the fault hypothesis $f_{j}\left(f_{y j}, f_{u j}, f_{a j}\right)$ and $t_{0}$ is the fault occurrence time instant. When $t_{0}$ is unknown, it must be estimated using the occurrence time instant $k_{\phi i}$ of the first observed fault signal $\phi_{i}(k)$. As consequence of the fault residual sensitivity time dependency, $\boldsymbol{F S M}$ sensit $t_{i j}$ evolves dynamically since the fault occurrence time instant $t_{0}$.

The consistency between the observed sequence of fault signals $\phi_{i}(k)$ and the theoretical information stored in FSMsensit for the $j^{\text {th }}$-fault hypothesis can be evaluated computing factorsensit ${ }_{j}$ as follows:

$$
\begin{gathered}
\text { factorsensit }_{j}(k)=\frac{\mid \sum_{i=1}^{n y}\left(\phi_{i}(k) \boldsymbol{F S M} \text { sensit }_{i j}\right) \mid}{\sum_{i=1}^{n y}\left|\boldsymbol{F S M s e n s i t} t_{i j}\right|} \boldsymbol{z} \boldsymbol{v} \boldsymbol{f}_{j} \\
\boldsymbol{w v f}_{j}=\left\{\begin{array}{c}
0, \quad \text { if } \exists i \in\{1, \ldots, n\} \text { with } \boldsymbol{F S M} \text { sensit }_{i j}=0 \\
\text { and }\left|\phi_{i}(k)\right| \geq 0.5 \\
1, \text { otherwise }
\end{array}\right.
\end{gathered}
$$

Thus, this factor uses the values $\phi_{i}(k)$ of every fault signal weighted by the corresponding elements FSMsensit $_{i j}$ related to the fault hypothesis $f_{j}$ in order to set the occurrence probability of this fault hypothesis. Thus, when computing factorsensit $_{j}$ such as it is indicated by (41), the following behaviour is obtained: those expected and observed fault signals support the fault hypothesis $\boldsymbol{f}_{j}$ while the observation of an unexpected fault signal let reject that fault hypothesis of the final diagnosis result. Moreover, those missing fault signals also affect indirectly the supportability of the fault hypothesis via the denominator of (41).

Concerning matrix FSM01, known as the binary theoretical fault signature matrix [12], it must be noticed this matrix can be easily derived from $\boldsymbol{F S M}$ sensit (40) applying the following conversion:

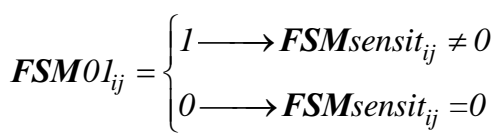

In this case, the consistency between the observed sequence of fault signals $\phi_{i}(k)$ and the theoretical information stored in FSM01 for the $j^{\text {th }}$-fault hypothesis can be evaluated computing factor $01_{j}$ as follows:

$$
\text { factor } 01_{j}(k)=\frac{\sum_{i=1}^{n y}\left(\text { boolean }\left(\phi_{i}(k)\right) \boldsymbol{F S M} 01_{i j}\right)}{\sum_{i=1}^{n y} \boldsymbol{F S M} 01_{i j}} \boldsymbol{z} \boldsymbol{f}_{j}
$$

with

$$
\operatorname{boolean}\left(\phi_{i}(k)\right)= \begin{cases}0, & \text { if }\left|\phi_{i}(k)\right|<0.5 \\ 1, & \text { if }\left|\phi_{i}(k)\right| \geq 0.5\end{cases}
$$

D. FSMtime: Evaluation of fault signal occurrence time instant

When a fault $f_{j}$ occurs, the affected residuals need different times to start indicating that fault or equivalently, the fault signals requires different times to appear. Each element of the $j^{\text {th }}$-column of the matrix FSMtime contains the time interval $\left[\underline{\varphi}_{i j}, \bar{\varphi}_{i j}\right]$ in which the fault signal $\phi_{i}(k)$ is expected to appear. The value $\bar{\varphi}_{i j}$ is associated with the minimum $f_{j}$-type fault which is considered to be isolated, while $\varphi_{i j}$ is associated with the maximum $f_{j}$-type fault the monitored system might suffer. Thus, the values $\underline{\varphi}_{i j}$ for a given fault $f_{j}$ could be estimated carrying out a test for every residual. This tests is based on comparing the residual disturbance caused by a fault in the nominal residual $r_{i}^{o}(k)$ with the adaptive threshold given by (23) related to the observer when the system is unaffected by a fault [21]. Derived from fault detection test (25) and from the residual internal form (31), this test can be written as:

$$
S_{r_{i}, f_{j}}\left(q^{-1}\right) f_{j}^{*}(k) q^{-t_{0}} \notin\left[\underline{r}_{i}^{o}(k), \bar{r}_{i}^{o}(k)\right] k \geq t_{0}
$$

where $f_{j}^{*}(k)$ is the worst case of a $f_{j}$-type fault the monitored system might suffer and $S_{r_{i}}, f_{j}$ is the sensitivity of the residual $r_{i}(k)$ regarding a fault $f_{j}(k)$. Then, the time the residual requires to start indicating the fault $\left(\delta_{i j}\right)$ is obtained using the minimum time instant $k_{\min }$ that satisfies (46).

$$
\delta_{i j}=k_{\min }-t_{0}
$$

When monitoring a system, the fault occurrence time instant $t_{0}$ is unknown in general. Hence, the values $\delta_{i j}$ associated to the fault hypothesis $f_{j}$ must be referred to the first observed fault signal. Then,

$$
\underline{\varphi}_{i j}=\underline{\delta}_{i j}-\min _{\forall i}\left(\underline{\delta}_{i j}\right)
$$

The value $\bar{\varphi}_{i j}$ might also be calculated using test (46) but in this case, $f_{j}^{*}(k)$ is the minimum $f_{j}$-type fault which is considered to be isolated. Thus, the values $\bar{\delta}_{i j}$ are obtained and then,

$$
\bar{\varphi}_{i j}=\bar{\delta}_{i j}-\min _{\forall i}\left(\underline{\delta}_{i j}\right)
$$

Regarding the elements of matrix FSMtime, 


$$
\text { FSMtime }_{i j}=\left\{\begin{array}{lll}
{\left[\underline{\varphi}_{i j}, \bar{\varphi}_{i j}\right]} & \text { if } & S_{r_{i}, f_{j}}\left(q^{-1}\right) \neq 0 \\
{[-1,-1]} & \text { if } & S_{r_{i}, f_{j}}\left(q^{-1}\right)=0
\end{array}\right.
$$

it is remarkable the influence of the observer gain on the interval $\left[\underline{\varphi}_{i j}, \bar{\varphi}_{i j}\right]$. Consequently, a proper design might help so that all the fault signals were observed at the same time instant.

Derived from FSMtime, one of the most important parameters of the fault isolation algorithm can be obtained. This is the time window $T_{w}$ which determines the maximum period of time required once the first fault signal is observed so that all fault signals can appear. In other words, $T_{w}$ is the period of time needed, once the first fault signal is detected, to give an accurate fault diagnosis result, unless there were only one fault hypothesis left supporting the observed fault signal temporal sequence before $T_{w}$ would have ended. Thereby, $T_{w}$ can be obtained as follows:

$$
T_{w}=\max _{\forall i, j}\left(\bar{\varphi}_{i j}\right)
$$

On the other hand, in order to compare the occurrence time instant of the observed fault signal sequence with the stored one in matrix FSMtime, the factor factortime $_{j}$ is calculated for every fault hypothesis as follows:

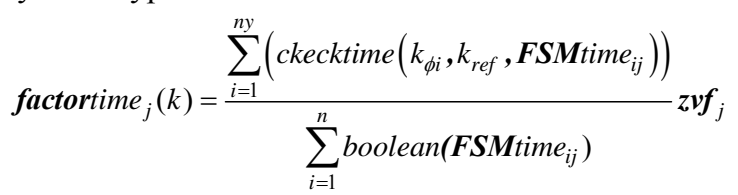

where $k_{\phi i}$ is the occurrence time instant of the fault signal $\phi_{i}(k), k_{\text {ref }}$ is the occurrence time instant of the first observed fault signal,

$$
\begin{aligned}
& \operatorname{ckecktime}\left(k_{\phi i}, k_{\text {ref }}, \boldsymbol{F S M} \text { time } e_{i j}\right)=
\end{aligned}
$$

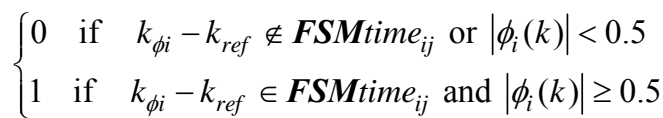

$$
\text { boolean } \left._{(\boldsymbol{F S M}} \text { time }_{i j}\right)= \begin{cases}0, & \text { if } \boldsymbol{F S M t i m e ~}_{i j}=[-1,-1] \\ 1, & \text { if } \boldsymbol{F S M t i m e ~}_{i j} \neq[-1,-1]\end{cases}
$$

Concerning the occurrence order property of the fault signals affected by the fault hypothesis $\boldsymbol{f}_{j}$, the $j^{\text {th }}$-column of the table FSMorder contains their theoretical occurrence order which is codified using ordinal numbers, starting with ' 1 '. Regarding those fault signals which are theoretically not affected by that fault hypothesis, they are coded with ' 0 ' in the corresponding cells of the matrix FSMorder. Thus, the elements of this matrix for a $f_{j}$-type fault can be derived from FSMtime applying the following rule:

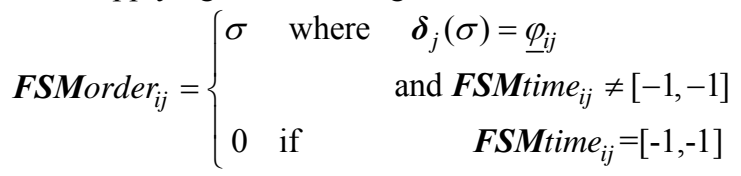

where $\delta_{j}$ is a vector that contains the non-repeated elements $\underline{\varphi}_{i j}$ of the $j^{\text {th }}$-column of FSMtime ordered ascendant and whose values are not equal to -1 . According to the residual internal form (31), it can be seen that the fault signal occurrence order for a given fault hypothesis is determined basically by the fault residual sensitivity function and by the adaptive threshold.

Then, comparing the fault signal observed information with the theoretical one stored in the matrix FSMorder, the occurrence probability of every fault hypothesis can be calculated computing factororder $_{j}$ :

$$
\text { factororder }_{j}(k)=\frac{\sum_{i=1}^{n y}\left(\operatorname{ckeckorder}\left(\phi_{i}(k), \boldsymbol{F S M} \text { Morder }_{i j}\right)\right)}{\left.\sum_{i=1}^{n y} \text { boolean }_{(\boldsymbol{F S M o r d e r}}{ }_{i j}\right)} \boldsymbol{z} \boldsymbol{v \boldsymbol { f } _ { j }}
$$

where

$$
\begin{aligned}
& \text { ckeckorder }\left(\phi_{i}(k), \boldsymbol{F S M o r d e r} r_{i j}\right)= \\
& \left\{\begin{array}{lll}
0 & \text { if } & \left|\phi_{i}(k)\right|<0.5 \\
0 & \text { if } & \operatorname{order}\left(\phi_{i}(k)\right) \neq \boldsymbol{F S M o r d e r} \\
1 & \text { if } & \operatorname{order}\left(\phi_{i}(k)\right)=\text { and }\left|\phi_{i}(k)\right| \geq 0.5
\end{array}\right.
\end{aligned}
$$

and $\operatorname{order}\left(\phi_{i}(k)\right)$ is the observed occurrence order of the fault signal $\phi_{i}(k)$.

\section{E. Application to the case study}

Considering the set of interval reduced observers (Section IV.D) used to monitor the limnimeter outputs of the Barcelona sewer network presented in Fig. 5, the value of the matrices FSMsensit and FSMtime are given in this section. These matrices are computed taking into account the observer gains $w_{i, j} \quad\left(\lambda_{i, j}=w_{i, j} a_{i, j}\right)$ of all interval observers are equal to 0.01 (Section IV.D) and the occurrence of the first fault signal is detected at time instant $t_{0}=4000 \mathrm{~s}$.

Regarding to FSMsensit (see Section V.C), it must be taken into account that each element of this matrix is a time function mainly based on the sensitivity of the residual related to a certain fault signal to a given fault hypothesis (40). Thus, in the following, the elements of $\boldsymbol{F S M}$ sensit matrix illustrated in Table II are just the fault residual sensitivity steady-state values instead of the ones derived from (40). However, the presented fault isolation algorithm does use this equation to obtain the elements of $\boldsymbol{F S M}$ sensit. In this table, $f_{L j}$ is a fault affecting the limnimeter $L_{j}$ while $\phi_{L i}$ is the fault signal associated with the residual $r_{L i}$ obtained using the interval observer model of $L_{i}$. For instance, considering the column of FSMsensit associated with the fault hypothesis $f_{L 4}$, the value of the cell related to the fault signal $\phi_{\mathrm{L} 4}$ is given by the steadystate value of the sensitivity function $S_{r_{4}, f_{L 4}}\left(q^{-1}\right)$ (37) when applied an unitary abrupt step input $\eta(k)$ at $t_{0}=4000 \mathrm{~s}$.

Table III presents FSMtime matrix (see Section V.D), where the fault occurrence time intervals are expressed in seconds. According to the value of the presented FSMtime and (51), the value of the diagnosis time window for this scenario is $T_{w}=30600 \mathrm{~s}$, taking into account that $T_{\mathrm{s}}=300 \mathrm{~s}$ is 
the sample time period.

TABLE II

THEORETICAL FAULT SIGNATURE MATRIX RELATED TO THE FAULT RESIDUAL SENSITIVITY PROPERTY, FSMsensit

\begin{tabular}{|c|c|c|c|c|c|c|c|c|c|c|c|c|c|c|c|}
\hline & $f_{L 1}$ & $f_{L 2}$ & $f_{L 3}$ & $f_{L 4}$ & $f_{L 5}$ & $f_{L 6}$ & $f_{L 7}$ & $f_{L 8}$ & $f_{L 9}$ & $f_{L 10}$ & $f_{L 11}$ & $f_{L 12}$ & $f_{L 13}$ & $f_{L 14}$ \\
\hline$\phi_{L 1}$ & 0.964 & 0 & 0 & 0 & 0 & 0 & 0 & 0 & 0 & 0 & 0 & 0 & 0 & 0 \\
\hline$\phi_{L 2}$ & -0.908 & 0.955 & 0 & 0 & 0 & 0 & 0 & 0 & 0 & 0 & 0 & 0 & 0 & 0 \\
\hline$\phi_{L 3}$ & 0 & 0 & 0.977 & 0 & 0 & 0 & 0 & 0 & 0 & 0 & 0 & -0.255 & 0 & 0 \\
\hline$\phi_{L 4}$ & 0 & 0 & 0 & 0.789 & 0 & 0 & 0 & 0 & 0 & 0 & 0 & 0 & 0 & 0 \\
\hline$\phi_{L 5}$ & 0 & 0 & -0.552 & 0 & 0.952 & & -0.406 & 0 & 0 & 0 & 0 & 0 & 0 & -5.162 \\
\hline$\phi_{L 6}$ & 0 & 0 & & -0.340 & 0 & 0.921 & 0 & 0 & 0 & 0 & 0 & 0 & 0 & 0 \\
\hline$\phi_{L 7}$ & 0 & 0 & 0 & 0 & 0 & 0 & 0.802 & 0 & 0 & 0 & 0 & 0 & 0 & 0 \\
\hline$\phi_{L 8}$ & 0 & 0 & 0 & 0 & 0 & 0 & 0 & 0.933 & 0 & 0 & 0 & 0 & -0.150 & 0 \\
\hline$\phi_{L 9}$ & 0 & 0 & 0 & 0 & 0 & 0 & 0 & 0 & 0.946 & 0 & 0 & 0 & 0 & 0 \\
\hline$\phi_{L 10}$ & 0 & -57.243 & 0 & 0 & 0 & 0 & 0 & 0 & 0 & 0.362 & 97.484 & 0 & 0 & 0 \\
\hline$\phi_{L 12}$ & 0 & 0 & 0 & 0 & 0 & 0 & 0 & 0 & 0 & 0 & -14.085 & 1 & 0 & 0 \\
\hline$\phi_{L 14}$ & 0 & 0 & 0 & 0 & 0 & 0 & -0.183 & 0 & 0 & 0 & 0 & 0 & 0.265 & 1 \\
\hline
\end{tabular}

TABLE III

THEORETICAL FAULT SIGNATURE MATRIX RELATED TO THE FAULT SIGNAL OCCURRENCE TIME INSTANT PROPERTY, FSMTIME

\begin{tabular}{|c|c|c|c|c|c|c|c|c|c|c|c|c|c|c|}
\hline & $f_{L 1}$ & $f_{L 2}$ & $f_{L 3}$ & $f_{L 4}$ & $f_{L 5}$ & $f_{L 6}$ & $f_{L 7}$ & $f_{L 8}$ & $f_{L g}$ & $f_{L 10}$ & $f_{L 11}$ & $f_{L 12}$ & $f_{L 13}$ & $f_{L 14}$ \\
\hline$\phi_{L 1}$ & {$[0,0]$} & {$[-1,-1]$} & {$[-1-1,-1]$} & {$[-1-1,-1]$} & {$[-1,-1]$} & {$[-1-1,-1]$} & {$[-1,-1]$} & {$[-1,-1]$} & {$[-1,-1]$} & {$[-1,-1]$} & {$[-1-1-1]$} & {$[-1-1-1]$} & {$[-1-1,-1]$} & {$[-1,-1]$} \\
\hline$\phi_{L 2}$ & {$\left[\begin{array}{c}{[300,3900} \\
]\end{array}\right]$} & {$[0,0]$} & {$[-1,-1]$} & {$[-1-1]$} & {$[-1,-1]$} & {$[-1,-1]$} & {$[-1,-1]$} & {$[-1,-1]$} & {$[-1,-1]$} & {$[-1,-1]$} & {$[-1-1]$} & {$[-1,-1]$} & {$[-1,-1]$} & {$[-1,-1]$} \\
\hline$\phi_{L 3}$ & {$[-1,-1]$} & {$[-1,-1]$} & {$[0,0]$} & {$[-1,-1]$} & {$[-1,-1]$} & {$[-1-1-1]$} & {$[-1,-1]$} & {$[-1,-1]$} & {$[-1,-1]$} & {$[-1,-1]$} & {$[-1,-1]$} & \begin{tabular}{|c}
300,2400 \\
1 \\
\end{tabular} & {$[-1,-1]$} & {$[-1,-1]$} \\
\hline$\phi_{L 4}$ & {$[-1,-1]$} & {$[-1,-1]$} & {$[-1,-1]$} & {$[0,0]$} & {$[-1,-1]$} & {$[-1,-1]$} & {$[-1,-1]$} & {$[-1,-1]$} & {$[-1,-1]$} & {$[-1,-1]$} & {$[-1-1,-1]$} & {$[-1-1,-1]$} & {$[-1,-1]$} & {$[-1,-1]$} \\
\hline$\phi_{L 5}$ & {$[-1,-1]$} & {$[-1,-1]$} & $\begin{aligned} 600,4200 \\
1\end{aligned}$ & {$[-1-1]$} & {$[0,0]$} & {$[-1,-1]$} & 900,4200 & {$[-1,-1]$} & {$[-1,-1]$} & {$[-1-1,-1]$} & {$[-1,-1]$} & {$[-1,-1]$} & {$[-1,-1]$} & 300,2400 \\
\hline$\phi_{L G}$ & {$[-1,-1]$} & {$[-1,-1]$} & {$[-1,-1]$} & 900,6300 & {$[-1,-1]$} & {$[0,0]$} & {$[-1,-1]$} & {$[-1,-1]$} & {$[-1,-1]$} & {$[-1,-1]$} & {$[-1,-1]$} & {$[-1-1]$} & {$[-1,-1]$} & {$[-1,-1]$} \\
\hline$\phi_{L 7}$ & {$[-1-1,-1]$} & {$[-1,-1]$} & {$[-1-1]$} & {$[-1-1,-1]$} & {$[-1,-1]$} & {$[-1-1-1]$} & {$[0,0]$} & {$[-1,-1]$} & {$[-1,-1]$} & {$[-1,-1]$} & {$[-1-1,-1]$} & {$[-1,-1]$} & {$[-1,-1]$} & {$[-1,-1]$} \\
\hline$\phi_{L B}$ & {$[-1,-1]$} & {$[-1,-1]$} & {$[-1-1,-1]$} & {$[-1,-1]$} & {$[-1,-1]$} & {$[-1-1]$} & {$[-1,-1]$} & {$[0,0]$} & {$[-1,-1]$} & {$[-1,-1]$} & {$[-1,-1]$} & {$[-1,-1]$} & 100,540 & {$[-1,-1]$} \\
\hline$\phi_{L 9}$ & {$[-1,-1]$} & {$[-1,-1]$} & {$[-1,-1]$} & {$[-1-1-1]$} & {$[-1,-1]$} & {$[-1,-1]$} & {$[-1-1,-1]$} & {$[-1,-1]$} & {$[0,0]$} & {$[-1,-1]$} & {$[-1,-1]$} & {$[-1,-1]$} & {$[-1,-1]$} & {$[-1,-1]$} \\
\hline$\phi_{L 10}$ & {$[-1,-1]$} & $\begin{array}{c}{[300,1800} \\
]\end{array}$ & {$[-1,-1]$} & {$[-1-1-1]$} & {$[-1,-1]$} & {$[-1,-1]$} & {$[-1,-1]$} & {$[-1,-1]$} & {$[-1,-1]$} & {$[0,0]$} & \begin{tabular}{|l|l|}
300,30600 \\
\end{tabular} & {$[-1-1]$} & {$[-1,-1]$} & {$[-1,-1]$} \\
\hline$\phi_{L 12}$ & {$[-1,-1]$} & {$[-1,-1]$} & {$[-1-1]$} & {$[-1,-1]$} & {$[-1,-1]$} & {$[-1-1]$} & {$[-1,-1]$} & {$[-1,-1]$} & {$[-1,-1]$} & {$[-1,-1]$} & {$[0,0]$} & {$[0,0]$} & {$[-1,-1]$} & {$[-1,-1]$} \\
\hline$\phi_{L 14}$ & {$[-1-1,-1]$} & {$[-1,-1]$} & {$[-1,-1]$} & {$[-1-1,-1]$} & {$[-1,-1]$} & {$[-1,-1]$} & {$[0,0]$} & {$[-1,-1]$} & {$[-1,-1]$} & {$[-1,-1]$} & {$[-1,-1]$} & {$[-1-1,-1]$} & {$[0,0]$} & {$[0,0]$} \\
\hline
\end{tabular}

\section{FAULt ISOLATION MODULE}

\section{A. Background}

The basic idea of a fault isolation module is that the occurrence of a fault will generate a unique sequence of observable fault signals (events) that will establish the presence of a given fault. In general, the model type (qualitative or quantitative) used in fault isolation depends on the type of the used fault detection model. However, since a fault signal can be seen as a discrete-time event with a given occurrence time instant, dynamics and duration, the use of those qualitative models known as timed discrete events models follows naturally [13][15]. In this sense, [9] uses a labelled transition system (LTS), considering the fault signal occurrence order. This LTS is built on the grounds of a causal graph that models the behaviour of the monitored system. Conversely, temporal dynamic table of states (T-DTS) method [14] models the relationship between fault signals and faults using the called Fault Information System (FIS). The fault isolation algorithm used by this method is based on series inference where the occurrence of a new fault signal let narrow the possible fault hypotheses checking its observed properties and the information stored in the FIS. However, this kind of models is not very common when fault detection stage is modeled using an analytical model (at least in the FDI community). In this paper, the proposed fault diagnosis approach will combine a fault isolation qualitative timed discrete event model with an analytical model used in fault detection.

\section{B. Fault Isolation Algorithm as a DES}

As mentioned before, the fault isolation module can be formalized as a discrete events system $(D E S)^{7}$ since a fault signal can be seen as a discrete-time event. Thereby, the occurrence of a fault will cause a sequence of fault signals whose dynamical properties should allow obtaining a fault diagnosis result. Taking into account temporal aspects in the sequence of fault signals (order and time instant of occurrence), a timed discrete event system (TDES) will allow to model more accurately the fault isolation process from the occurrence of the first fault signal of the temporal sequence until a fault isolation result is given. A timed discrete event system of this type is known as a timed labeled transition system (TLTS). A TLTS can be seen as an evolution of the labeled transition system family (LTS) [9] since a LTS does not consider the occurrence time instant of the fault signals in order to determine the fault isolation result. Using this modelling approach, the fault signals would be the events, the states would be given by all the fault hypotheses supporting the observed fault signal sequence and the transitions would be set by the comparison between the theoretical and the observed dynamical properties of the fault signals.

\section{Fault Isolation and Interface Module Components}

1) Description: Fig. 8 presents the components of the fault isolation and interface modules which derive from an evolution of the architecture proposed in [28]. As mentioned above, the main idea of this fault isolation process is that a given fault affecting the monitored system will cause a unique temporal sequence of fault signals which will allow obtaining a diagnosis result comparing their observed dynamical properties with the ones stored for each fault hypothesis in the fault isolation matrices.

The fault isolation algorithm starts with the occurrence of the first fault signal and ends when there is only one fault hypothesis supporting the observed temporal sequence of fault signals or when the diagnosis time window $T_{w}(51)$ has ended. Thereby, the first element of this algorithm is a memory component which registers some information of the observed fault signals. The second element is a timed series inference component which compares the stored information of a new observed fault signal with the information stored in matrices FSM01, FSMtime, and FSMorder for those non-rejected fault hypotheses. The result of this series inference component is the rejection of those fault hypotheses that do not support the observations. When there is only one fault hypothesis left, the algorithm ends giving that hypothesis as the fault diagnostic

\footnotetext{
${ }^{7}$ Fault isolation methods built using a DES consist of a set of states connected by transitions [34]. The transitions are related to events generated by the fault effect on the monitored system, while the states indicate a certain situation of the whole fault isolation process. Moreover, when these transitions are built taking into account some temporal aspects, the discrete event system (DES) is known as a timed discrete event system (TDES) [6].
} 
result. Otherwise, when the time window $T_{w}$ has ended, the third element, the pattern comparison component, computes factorsensit ${ }_{j}$ for those non-rejected fault hypotheses. Then, the last element, the logic decision component, gives as a diagnostic result the fault hypothesis with the biggest absolute value of the factor factorsensit ${ }_{j}$.

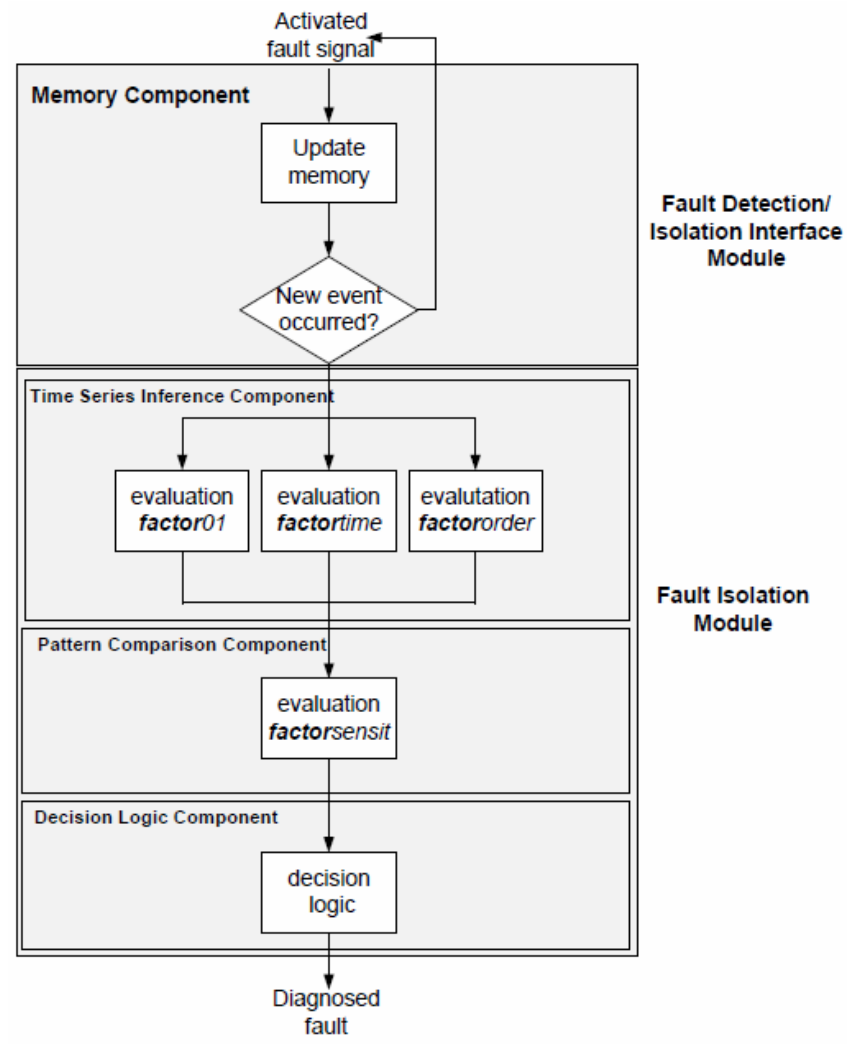

Fig. 8. Components of Interface and Fault Isolation Modules

In the following, a more detailed explanation of the performance of the memory component and the timed series inference component will be given.

2) Memory Component: The memory component consists in a table which stores some information related to the time evolution of each fault signal. Thereby, once the first fault signal is observed the memory component stores the occurrence time instant $\left(k_{\phi i}\right)$, defined as the first time instant where $\phi_{i}\left(k_{\phi i}\right)$ is activat $\mathrm{d}_{\mathrm{e}} \mathrm{a}$ the fault signal value $\left(\phi_{\text {imax }}\right)^{8}$ whose absol $_{\text {ute }}$ value is maximum. Every time the fault detection module detects a new fault signal $\phi_{i}(k)$ or an observed fault signal reaches a new value $\phi_{\text {imax }}$, this memory is updated with the new information situation. Then, after a fault diagnosis result is given by the fault isolation module, the memory component is reset being ready to start the diagnosis of a new fault. This reset consists of deleting the information related to all vanished fault signals and the value

$$
{ }^{8} \phi_{i \max }=\phi_{i}\left(k_{i \max }\right) \text { where }\left|\phi_{i \max }\right|=\max \underset{k \geq k_{\phi i p}}{ }\left(\left|\phi_{i}(k)\right|\right)
$$

$\phi_{\text {imax }}$ of those fault signals which can still be observed at this time instant.

3) Timed series inference component: This component is based on the fact that each new fault signal allows rejecting those hypotheses that do not support the observations. In consequence, a diagnosis result can be given before the time window $T_{w}$ ends. Thereby, the rejection of a certain fault hypothesis when a new fault signal is observed is based on the comparison of its information stored in the memory component with its theoretical one for this fault hypothesis stored in the matrices FSM01, FSMtime, and FSMorder. This leads to the following performance: the observation of a new fault signal will allow narrowing the subset of fault hypotheses which are still supporting the observations and consequently, the ones which are still candidates to set the diagnosis result. When there is just one fault hypothesis left, the reasoning process ends giving it as the diagnosis result. Otherwise, the process ends once the period of time $T_{w}$ has ended since the observation of the first fault signal.

This component can be built using a timed labelled transition system where the initial state is the non-faulty state, then, each fault hypotheses ( $\operatorname{set} f$ ) have a TLTS representation which are connected to this initial state. The TLTS representation associated with a given fault hypothesis shows the fault signal temporal sequence caused by this fault. In each state transition, the properties of the new observed fault signal are compared with those stored in FSM01, FSMorder and/or FSMtime for this fault hypothesis. The present state of a fault hypothesis TLTS representation just indicates that this fault hypothesis is still supporting the observed fault signal temporal sequence. When a new fault signal occurs, for each non-rejected fault hypotheses, the state transition starting at the present state is evaluated. If this evaluation fails, the fault hypothesis is rejected. At the end of the diagnosis time window $T_{w}$, those non-rejected fault hypotheses will establish the final fault diagnosis result.

According to the definition given by [9] for a LTS, a TLTS can be seen as the following tuple

$$
\mathcal{F}_{j}=\left(Q_{j}, q_{0}, \boldsymbol{\Sigma}_{j}, \boldsymbol{\delta}_{j}\right)
$$

where $Q_{j}$ is the set of states, $q_{0}$ is the initial state, $\Sigma_{j}$ is the set of labels and $\boldsymbol{\delta}_{j}$ is the set of transitions. Thus, when applying this TLTS definition to model the presented timed series inference component, there will be a tuple $\mathcal{T}_{j}$ related to every fault hypothesis $f_{j}$ that belongs to the set $\boldsymbol{f}$ of all the considered fault hypotheses. All this set of elements $\mathcal{T}_{j}$ will just have one component in common: the state $q_{0}$ related to the non-faulty state. Regarding the states $Q_{p j} \in Q_{j}$, its number $n_{Q j}$ is set by the number of fault signals $\phi_{i}(k)$ affected by the fault hypothesis $f_{j}$. This is

$$
n_{Q j}=\sum_{i=1}^{n y} \boldsymbol{F S M} 01_{i j}
$$


The present state $Q_{p j}$ of $\mathcal{T}_{j}$ just have the meaning that the fault hypothesis $f_{j}$ is still supporting the observations.

Regarding the transitions $\delta_{p j}$ of the set $\delta_{j}$, they connect $Q_{(p-}$ 1)j with $Q_{p j}$ being $q_{0}$, the first state. Thus, there will be for a certain $\mathcal{T}_{j}$, a transition for every fault signal related to the fault hypothesis $f_{j}$ : this is $n_{Q j}$. In this way, the transition $\delta_{p j}$ will be related to the fault signal $\phi_{i}(k)$ whose theoretical occurrence order for this fault hypothesis is given by ' $p$ ' $\left(\boldsymbol{F S M o r d e r}_{i j}=p\right)$. Thereby, concerning the sequence of transitions and states in $\mathcal{T}_{j}$, they have an ascendant order set by $p=1, \ldots, n_{Q j}$.

About the labels $\boldsymbol{\Sigma}_{p j}$ of the set $\boldsymbol{\Sigma}_{j}$, there is one for each transition $\delta_{p j}$ and consequently, for each fault signal $\phi_{i}(k)$ related to the fault hypothesis $f_{j}$. Thereby, the evaluation of all the labels $\boldsymbol{\Sigma}_{p j}$ is carried out when the $p^{\text {th }}$-fault signal $\phi_{i}(k)$ is observed. If the evaluation of $\boldsymbol{\Sigma}_{\boldsymbol{p} j}$ fails, the fault hypothesis $f_{j}$ is rejected. Otherwise, the state $Q_{p j}$ becomes the present state of $\mathcal{T}_{j}$. Thereby, $\Sigma_{p j}$ is carried out evaluating the following relation

$$
\begin{gathered}
\sum_{p j}=\left(\operatorname{ckeckorder}_{\left.\left(\phi_{i}(k), \text { FSMorder }_{i j}\right)\right) \text { and }}\right. \\
\left(\operatorname{ckecktime}\left(k_{\phi i}, \boldsymbol{k}_{\text {ref }}, \text { FSMtime }_{i j}\right)\right)
\end{gathered}
$$

where the functions checkorder and checktime are given by (57) and (53), respectively. According to the mentioned previously, a diagram of the set of elements $\mathcal{T}_{j}$ which models the performance of this timed series inference component is presented in Fig. 9.

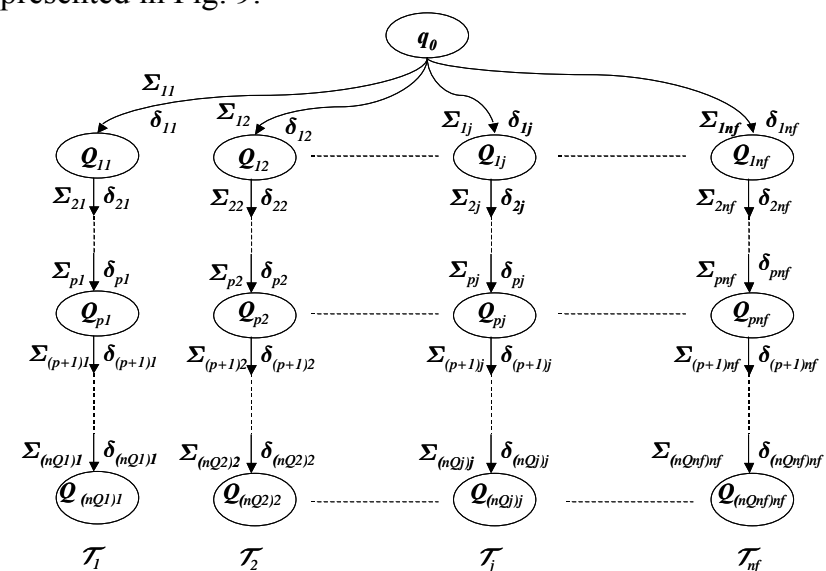

Fig. 9. Timed series inference component modelled using a timed labelled transition system

\section{Application to the Case Study}

In this section and for the considered case of study (see Fig. 5), a fault isolation process will be designed following the architecture presented in Section VI.C. The main focus will be on the timed labelled transition system used to model the timed series inference component (see Fig. 9). In this case, this fault isolation model will be built just considering a subset of 7 fault hypotheses affecting $L_{3}, L_{4}, L_{5}, L_{6}, L_{7}, L_{13}$ and $L_{14}$ of the fault set $f_{\boldsymbol{y}}$ (4). In Fig. 10, the label of the transition related to the fault signal $\phi_{L i}$ for the fault hypothesis $f_{L j}$ (fault affecting limnimeter $L_{j}$ ) will be noted as $\boldsymbol{\Sigma}_{L i-L j}(60)$ while all the states related to a certain fault hypothesis will be notated as $f_{L j}$.

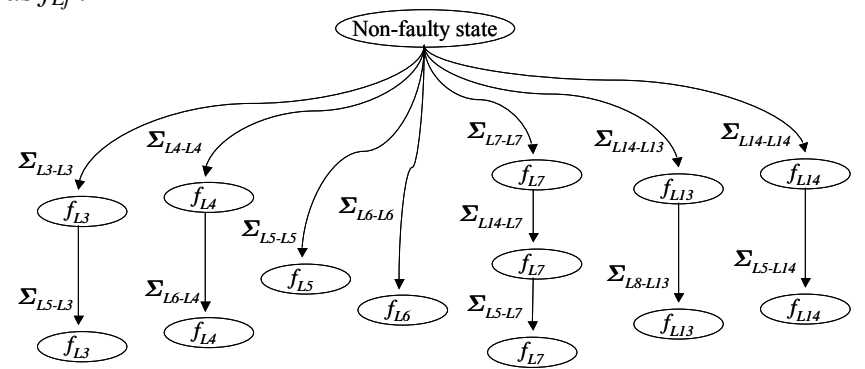

Fig. 10. Limnimeter fault isolation based on a timed LTS

The associated representation of this timed labelled transition system can be seen as the integration of all the information stored in FSM01, FSMorder and FSMtime in the same structure.

Focusing in a fault scenario ${ }^{9}$ where a fault affecting limnimeter $L_{4}$ occurs at $t_{0}=4000 \mathrm{~s}$, the time evolution of the affected residuals, $r_{4}^{o}(k)$ and $r_{6}^{o}(k)$ (34) and (35) and their associated adaptive thresholds $\left(\left[\underline{r}_{4}^{o}(k), r_{4}^{o}(k)\right] \quad\right.$ and $\left.\left[\underline{r}_{6}^{o}(k), \bar{r}_{6}^{o}(k)\right]\right)$ are plotted using the observer gains corresponding to $w_{44}=w_{66}=0.01$ ( Fig. 11).

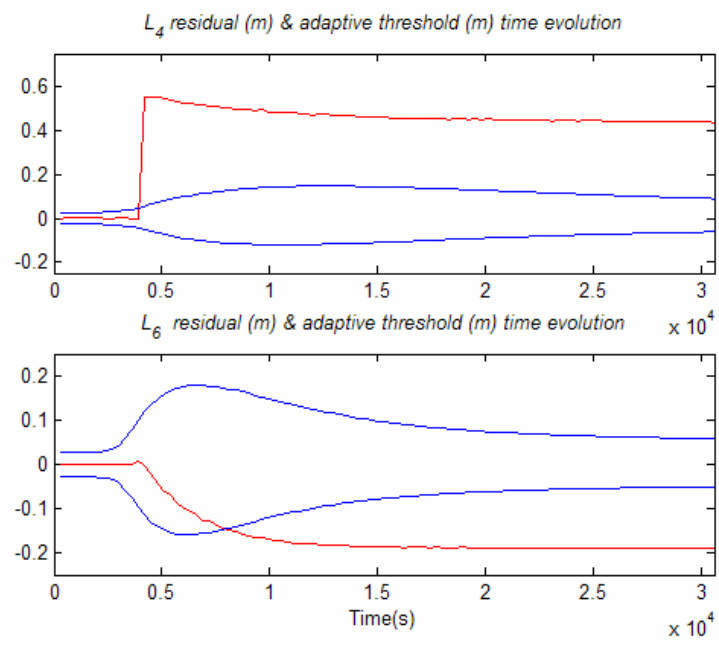

Fig. 11. Time evolution of the residuals and their adaptive thresholds.

Analyzing the time evolution of those residuals and according (25) and (26), the first observed fault signal will be $\phi_{\mathrm{L} 4}$ (fault signal related to limnimeter $L_{4}$ observer model)

\footnotetext{
${ }^{9}$ All faults scenarios presented in this paper have been simulated using a high-fidelity simulator of the Barcelona sewer network.
} 
since time instant $t=t_{0}$. The time evolution of factorsensit ${ }_{j}$ (41) and factortime f $_{j}$ (52) related to all fault hypotheses of the set $\boldsymbol{f}_{\boldsymbol{y}}(4)$ is plotted at every time instant in Fig. 12.
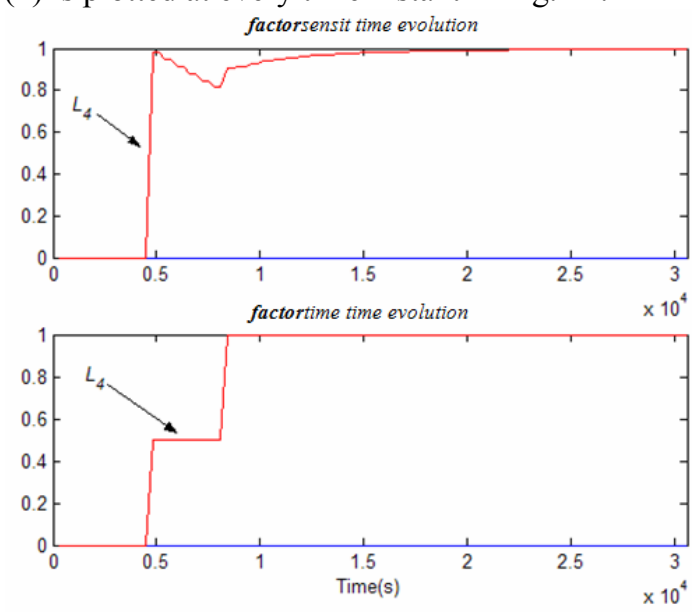

Fig. 12. Time evolution of factorsensit $t_{j}$ and factortime $_{j}$ related to all fault hypotheses of the set $f_{y}$.

It can be seen that only those factors related to the fault hypothesis $f_{L 4}$ are activated from the fault occurrence time instant. Then, according to the fault isolation discrete-event model presented Fig. 10 and the information stored in FSM01, FSMorder and FSMtime, all fault hypotheses except $f_{L 4}$ (fault affecting $L_{4}$ ) will be rejected. Afterwards, the fault signal $\phi_{L 6}$ is observed supporting the LTS representation associated with $f_{L 4}$ (see Fig. 13).

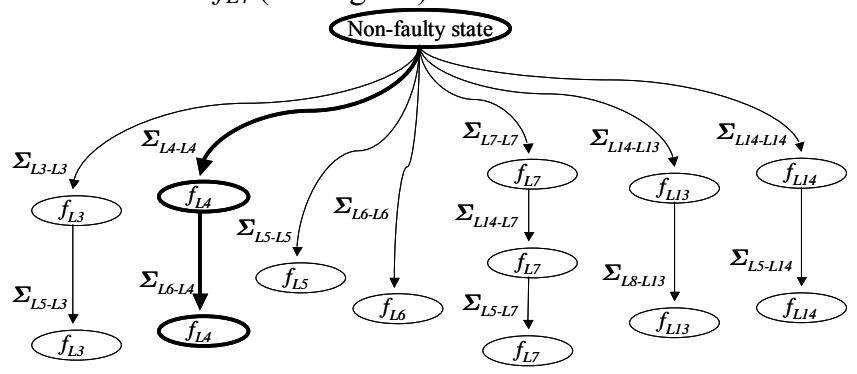

Fig. 13. Isolation of a fault affecting $L_{4}$ using a Timed LTS model.

\section{COMPARISON WITH STANDARD FDI APPROACH}

In this section, the proposed timed discrete-event FDI approach is compared with the standard FDI approach based on the binary diagnostic matrix (FSM01) [12] using a parallel diagnostic inference [12]. Thereby, this method tries to give a diagnosis result at every time instant checking the binary property of the observed fault signals with the theoretical information stored in matrix FSM01. Those fault hypotheses whose theoretical fault signature fully matches with the observed fault signals are given as a fault diagnosis result.

In the considered fault scenario, a fault affecting the limnimeter $L_{7}$ occurs at $t_{0}=4000 \mathrm{~s}$. In this case, all the interval observers used to monitor the limnimeters were tuned using the observer gains $\left(\lambda_{i, j}=w_{i, j} a_{i, j}\right)$ corresponding to $w_{i, j}=0.01$ except the one used to monitor $L_{7}$ whose observer gain was determined by $w_{7,7}=0.5$. In Fig. 14 , the time evolution of the affected residuals $\left(r_{5}^{o}(k), r_{7}^{o}(k), r_{8}^{o}(k), r_{14}^{o}(k)\right)$ and their adaptive thresholds are plotted. In this figure, for every residual, a fault detection indicator is plotted to signal out when the associated fault signal is observed or not (25).

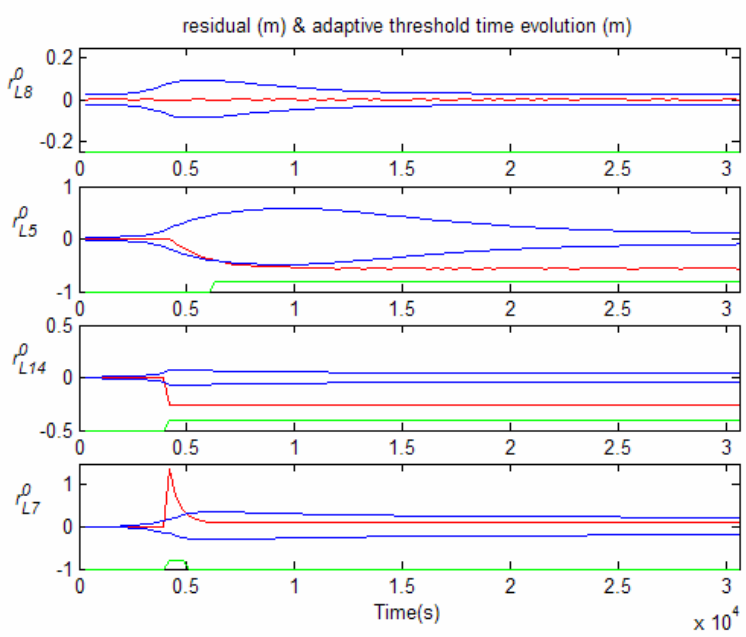

Fig. 14. Time evolution of the residuals, their adaptive thresholds and the associated fault detection indicator.

According to the matrix FSMsensit (Table II) and FSMtime (Table III), a fault affecting $L_{7}$ will cause the observation of three fault signals: $\phi_{L 7}$ and $\phi_{L 14}$ when the fault occurs and $\phi_{L 5}$ once some time has elapsed. Thus, in this scenario $\phi_{L 7}$ was the first observed fault signal. Moreover, due to the configuration of the observer that monitors $L_{7}, \phi_{L 7}$ is not observed persistently and vanishes before $\phi_{L 5}$ appears. In this situation, once $\phi_{L 7}$ has vanished, the binary fault signature associated with $f_{L 14}$ matches the binary property of the observed fault signals. In this case, the binary fault isolation approach will give $f_{L 14}$ as a fault diagnosis result instead of $f_{L 7}$, which is the right result.

In the following, the time evolution of the binary approach fault diagnosis result is given considering a set of possible results determined by $f_{L 3}, f_{L 5}, f_{L 7}, f_{L 13}, f_{L 14}$.

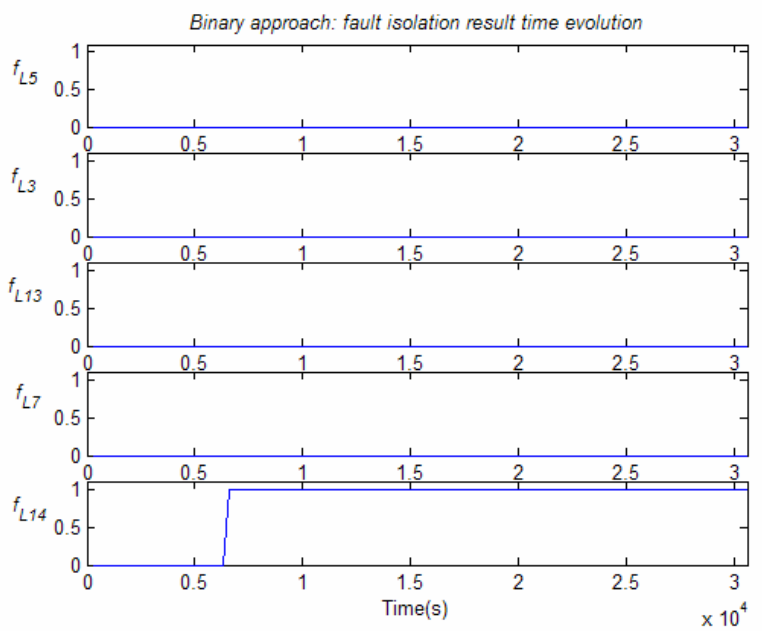

Fig. 15. Time evolution of the fault isolation result given by the binary approach. 
Fig. 15 shows how the binary approach gives a wrong fault diagnosis result as a consequence of the lack of persistence of $\phi_{\mathrm{L} 7}$

In Fig. 16 and Fig. 17, the time evolution of factorsensit $_{j}$ (41) and factortime $_{j}(52)$ related to the fault hypotheses $f_{L 3}$, $f_{L 5}, f_{L 7}, f_{L 13}, f_{L 14}$ is plotted at every time instant.

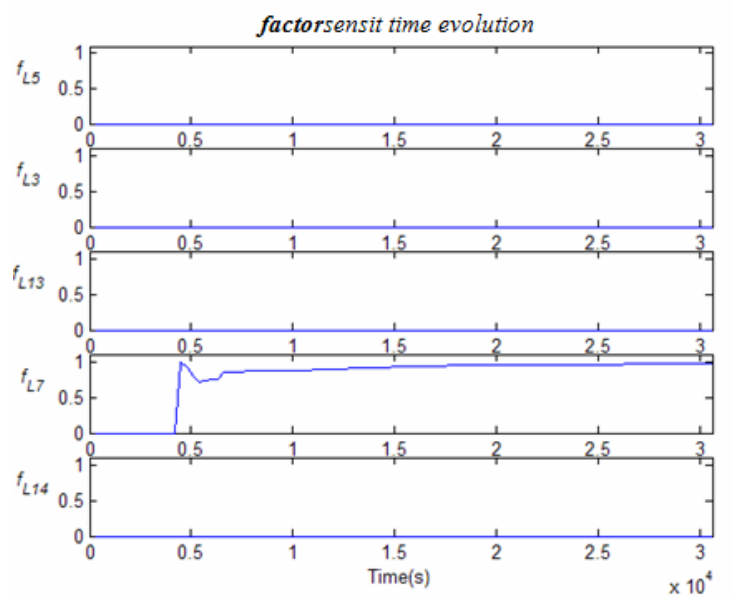

Fig. 16. Time evolution of factorsensit $t_{\mathrm{j}}$ related to the fault hypotheses $f_{L 3}, f_{L 5}$, $f_{L 7}, f_{L 13}, f_{L 14}$.

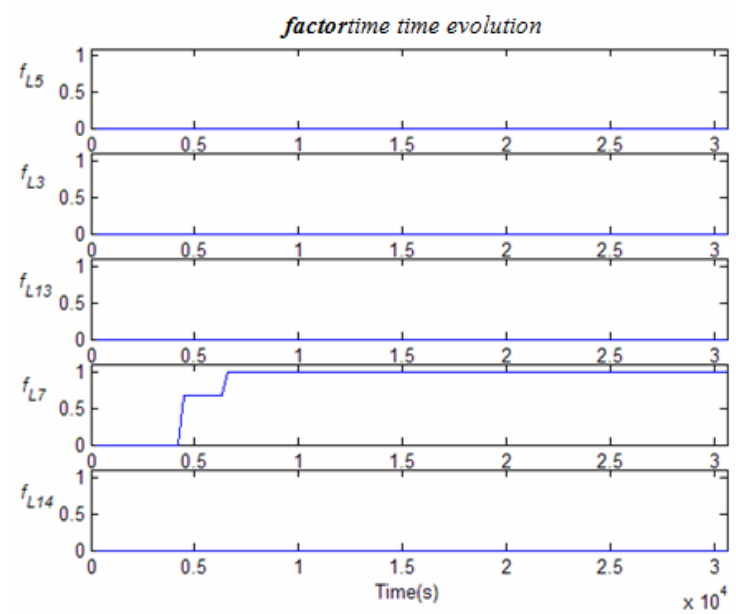

Figure 17. Time evolution of factortime $e_{j}$ related to the fault hypotheses $f_{L 3}, f_{L 5}$, $f_{L 7}, f_{L 13}, f_{L 14}$.

It can be seen that in spite of the lack of persistence of $\phi_{L 7}$, the proposed fault diagnosis method is not confused due to the used timed discrete event approach that takes into account the dynamical properties of the fault signals.

Then, according to the fault isolation discrete-event model presented Fig. 10 and the information stored in FSM01, FSMorder and FSMtime, all fault hypotheses except $f_{L 7}$ (fault affecting $L_{7}$ ) will be rejected (see Fig. 18).

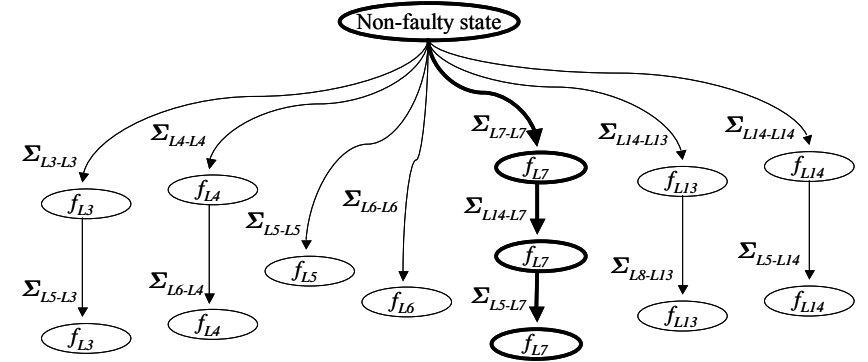

Fig. 18. Isolation of a fault affecting $L_{7}$ using a Timed LTS.

\section{CONCLUSIONS}

Taking into account the importance of fault detection and fault isolation on the optimal control of the Barcelona sewer network, this paper proposes a model-based fault diagnosis method using a timed discrete-event approach based on interval observers which improves the integration of fault detection and isolation tasks. This proposed approach tackles the drawbacks of the classical FDI fault diagnosis methods based on a binary interface between fault detection and fault isolation without considering the fault signal dynamics. As a consequence, when applying these classical methodologies to diagnose faults affecting the limnimeters of Barcelona sewer network, a poor performance of the control system was obtained. Concerning the proposed discrete event fault diagnosis approach, the interface between fault detection and fault isolation module considers the degree of fault signal activation and the occurrence time instant of the fault signals using a combination of several fault signature matrices which store the knowledge of the relationship between diagnostic signals and faults. Such fault signatures matrices can be derived from the system model using the fault sensitivity analysis. Moreover, exploiting the discrete-time event nature of the fault signals generated by the fault detection module, a fault diagnoser based on a timed discrete-event model can automatically be implemented. Using such approach, faults can be diagnosed since their occurrence generates a unique sequence of observable events (fault signals) that can be recognized by the isolation module. The states and transitions that characterize such a model can be inferred directly from the relationship between fault signals and faults. As a further research the method would be extended to handle multiplicative and multiple faults. Also the extension to nonlinear systems would be considered.

\section{REFERENCES}

[1] G. Biswas, M. O.Cordier, J. Lunze, L. Trave-Massuyes, M. Staroswiecki, "Diagnosis of Complex Systems: Bridging the Methodologies of the FDI and DX Communities," Systems, Man, and Cybernetics, Part B, IEEE Transactions on, vol. 34, no. 5, pp. $2159-$ 2162, Oct. 2004

[2] A. Boukhris, S. Giuliani, and G. Mourot, "Rainfall-runoff modeling for sensor diagnosis, " in Proc. IFAC SAFEPROCESS'97, Hull, England.,1997. 
[3] A. Boukhris, S. Giuliani, and G., Mourot, "Rainfall-runoff multimodelling for sensor fault diagnosis, " Control Engineering Practice, vol 9, no. 6, pp. 659-671, Jun. 2001.

[4] G. Cembrano, J. Quevedo, M. Salamero, V. Puig, J. Figueras, and J. Martí, "Optimal control of urban drainage systems: a case study," Control Engineering Practice, vol. 12, no. 1, pp. 1-9, Jan. 2004

[5] C. Combastel, S. Gentil, and J. P. Rognon, "Toward a better integration of residual generation and diagnostic decision," In Proc. IFAC SAFEPROCESS'03, Washington, USA.

[6] J. Chen, and R.J. Patton, "Robust Model-Based Fault Diagnosis for Dynamic Systems". Kluwer Academic Publishers, 1999.

[7] Y. L. Chen, and G. Provan, "Modeling and diagnosis of timed discrete event systems: a factory automation example," in Proc. of the American Control Conference, June, pp. 31-36, 1997.

[8] E. Chow, and A. Willsky,"Analytical redundancy and the design of robust failure detection systems," IEEE Transactions on Automatic Control, vol. 29 , no. 7 , pp. $603-614$, jul. 1984.

[9] M. Daigle, X. Koutsoukus, and G. Biswas, "A Discrete Event Approach to Diagnosis of Continuous Systems" in Proc. Workshop on Principles of Diagnosis DX'07.,Nashville, TN, USA, 2007.

[10] S. Duchesne, A. Mailhot, E. Dequidt, and J. Villeneuve, "Mathematical modeling of sewers under surcharge for real time control of combined sewer overflows". Urban Water, vol. 3, no. 4, pp.241-252, Dec. 2001.

[11] J. Figueras, G. Cembrano, V. Puig, J. Quevedo, M. Salamero, and J. Martí, "CORAL: an object-oriented tool for optimal control of sewer networks,". in proc. IEEE International Symposium on computer aided control system design, pp. 224-229, 2002.

[12] J. Gertler, Fault Detection and Diagnosis in Engineering Systems, M. Dekker, 1998

[13] M. W. Hofbaur, and B. C. Williams, "Hybrid estimation of complex systems," IEEE Transactions on Systems, Man, and Cybernetics, Part B, vol. 34, no. 5, pp. $2178-2191$, Oct. 2004.

[14] J.M. Kościelny, J. Korbicz, Z. Kowalczuk, and W. Cholewa, Fault Diagnosis. Springer-Verlag, Chapter 18, 2004.

[15] J. Luo, M. Namburu, K.R. Pattipati, L. Qiao and and Chigusa, S. "Integrated Model-Based and Data-Driven Diagnosis of Automotive Antilock Braking Systems". IEEE Transactions on Systems, Man, and Cybernetics, Part A: Systems and Humans, Vol. 40 (2), pp. $321-336$, 2010.

[16] J. Lunze, P. Supavatanakul, V. Puig, J. Quevedo, "Diagnosis of timed automata: Theory and application to the DAMADICS actuator benchmark problem" Control Engineering Practice vol. 14, no. 6, pp. 609-619, Jun. 2006

[17] M. Marinaki, and M. Papageorgiou, Optimal Real-time Control of Sewer Networks, Springe, 2005.

[18] J. Meseguer, V. Puig, T. Escobet, and J. Quevedo, "Observer gain effect in linear interval observer-based fault isolation" in proc. Workshop on Principles of Diagnosis DX'06, Peñaranda de Duero, Spain, 2006.

[19] J. Meseguer, V. Puig, and T. Escobet, "Observer gain effect in linear interval observer-based fault detection," in prod. IFAC SAFEPROCESS'06, Beijing, China, 2006.

[20] J. Meseguer, V. Puig, T. Escobet, and J. Quevedo, "Sensor Fault Diagnosis using Linear Interval Observers" in proc.Workshop on Principles of Diagnosis DX'07. Nashville, TN, USA, 2007.

[21] J. Meseguer, V. Puig, and T. Escobet, "Observer Gain Effect in Linear Interval Observer-based Fault Detection" in proc. IEEE Conference on Decision and Control CDC'07, New Orleans, USA, 2007.

[22] S. Narasimhan, and G. Biswas, "Model-Based Diagnosis of Hybrid Systems," IEEE Transactions on Systems, Man and Cybernetics, Part A, vol. 37, no. 3, pp. 348 - 361, May 2007.

[23] R.J. Patton, P. M. Frank, and R.N. Clark. Issues of fault diagnosis for dynamics systems, Springer. 2000

[24] T. F. Petti, J. Klein, and P. S. Dhurjati, "Diagnostic model processor: Using deep knowledge for process fault diagnosis," AIChE Journal, vol. 36, no. 4, pp. 565-575, Jan, 1990.

[25] S. Ploix, O. Adrot, and J. Ragot, "Parameter Uncertainty Computation in Static Linear Models," in proc. 38th IEEE Conference on Decision and Control, Phoenix, Arizona, USA, 1999.

[26] V. Puig, J. Saludes, J. Quevedo, "Worst-Case Simulation of Discrete Linear Time-Invariant Dynamic Systems", Reliable Computing, vol. 9, no.4, pp. 251-290, Aug. 2003.

[27] V. Puig, J. Quevedo, T. Escobet, and B. Pulido, "On the integration of fault detection and isolation in model based fault diagnosis" in proc.
Workshop on Principles of Diagnosis DX'04. Carcassonne, France, 2004.

[28] V. Puig, F. Schmid, J. Quevedo, and B. Pulido. "A New Fault Diagnosis Algorithm that Improves the Integration of Fault Detection and Isolation" in proc. ECC-CDC'05, Sevilla, Spain, 2005.

[29] V. Puig, J. Quevedo, T. Escobet, F. Nejjari, and S. de las Heras, "Passive Robust Fault Detection of Dynamic Processes Using Interval Models", IEEE Transactions on Control Systems Technology, Vol. 16, no. 5, pp. 1083 - 1089, Sep. 2008.

[30] V. Puig, "Fault Detection and Isolation in Sewer Networks". In proc. IFAC SAFEPROCESS'2009. Barcelona. Spain, 2009.

[31] V. Puig, G. Cembrano, J. Romera, J, Quevedo, B. Aznar, G. Ramón, and J. Cabot, "Predictive optimal control of sewer networks using CORAL tool: application to Riera Blanca catchment in Barcelona"; Water Sci Technol, vol. 60, no. 4, pp.:869-78, 2009

[32] B. Pulido, V. Puig, T. Escobet, and J. Quevedo, "A new fault localization algorithm that improves the integration between fault detection and localization in dynamic systems," in proc. Workshop on Principles of Diagnosis DX'05. Monterey, California, USA, 2005.

[33] M. Rodrigues, D. Theilliol, M. Adam-Medina, D. Sauter, "A fault detection and isolation scheme for industrial systems based on multiple operating models," Control Engineering Practice, vol..16, no. 2, pp. 225-239, Feb. 2008,

[34] M. Sampath, R. Sengupta, S. Lafortune, K. Sinnamohideen, and D. Teneketzis, "Failure diagnosis using discrete-event models, IEEE Transactions on Control Systems Technology, vol. 4, no. 2, pp. 105-124, 1996.

[35] S. Singh, A. Kodali, K. Choi, K. R. Pattipati, S. M. Namburu, S. C. Sean, D. V. Prokhorov, and L. Qiao, "Dynamic Multiple Fault Diagnosis: Mathematical Formulations and Solution Techniques," IEEE Transactions on Systems, Man and Cybernetics, Part A, vol. 39, no. 1, pp. $160-176$, Jan. 2009

[36] D. Theilliol, D. Sauter, L. G. Vela Valdes, « Integration of Qualitative and Quantitative Methods for Fault Detection Isolation," In proc. of IFAC SAFEPROCESS'97, Hull, UK, 1997

[37] L. Travé-Massuyès, T. Escobet, X. Olive, "Diagnosability Analysis Based on Component-Supported Analytical Redundancy Relations, “ IEEE Transactions on Systems, Man and Cybernetics, Part A, IEEE, vol. 36 , no. 6, pp. 1146 - 1160, Nov. 2006

[38] G. J. G. Upton and A. R. Rahimi (2003) "On-line detection of errors in tipping-bucket raingauges”, Journal of Hydrology, vol. 278, no.1-4, pp. 197-212, July 2003.

[39] M. Krysander and E. Frisk. "Sensor Placement for Fault Diagnosis". IEEE Transactions on Systems, Man, and Cybernetics, Part A: Systems and Humans, Vol. 38 (6), pp. 1398 - 1410, Nov. 2008.

[40] M. Krysander, J. Åslund, and M. Nyberg. "An Efficient Algorithm for Finding Minimal Overconstrained Subsystems for Model-Based Diagnosis". IEEE Transactions on Systems, Man, and Cybernetics, Part A: Systems and Humans, Vol. 38 (1), pp. 197 - 206, Jan. 2008.

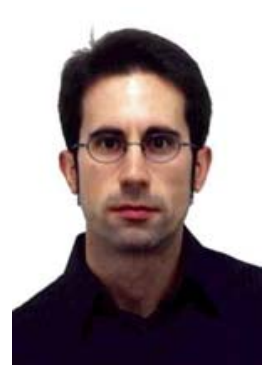

Jordi Meseguer received the Ph.D. Degree in Control Engineering in 2009 and the Industrial Engineering Degree in 1997, both from the Universitat Politècnica de Catalunya, Barcelona, Spain. He is currently an IT Analyst at CETaqua (Water Technological Centre, AGBAR Group) and a Research Member with the Advanced Control Systems research group at the Universitat Politècnica de Catalunya. His main research interests are focus on fault detection and isolation in complex industrial dynamic systems. He has published several papers in international conference proceedings and scientific journals. 


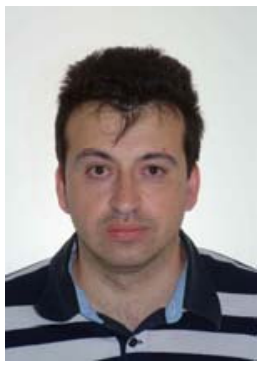

Vicenç Puig received the Ph.D. Degree in Control Engineering in 1999 and the Telecommunications Engineering Degree in 1993, both from the Universitat Politècnica de Catalunya, Barcelona, Spain. He is currently an Associate Professor of Automatic Control and leader of the Advanced Control Systems research group at the Universitat Politècnica de Catalunya. His main research interests are fault detection, isolation of faulttolerant control of dynamic systems. He has been involved in several European projects and networks and has published several papers in international conference proceedings and scientific journals.

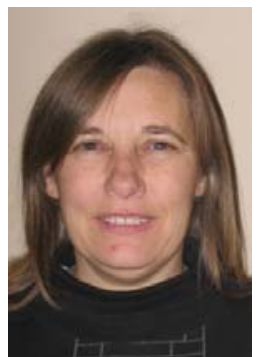

Teresa Escobet received the $\mathrm{Ph} . \mathrm{D}$. degree in industrial engineering in 1997 and the Industrial Engineering Degree in 1989, both from the Universitat Politècnica de Catalunya, Barcelona, Spain. She is currently an Associate Professor with the Universitat Politècnica de Catalunya, Catalonia, Terrassa, Spain, and a Lecturer in automatic control at Escola Politècnica Superior de Manresa, Spain. She is Research Member with the Advanced Control Systems research group of the Automatic Contro Department in Universitat Politècnica de Catalunya. Her main research interests are in dynamic system modeling and identification applied to fault detection, isolation, fault-tolerant control and condition-based maintenance. She has been involved in several European projects and networks and has published several papers in international conference proceedings and scientific journals. 